\title{
Report of the
}

\section{AD HOC PANEL ON HEAVY ION FACILITIES}

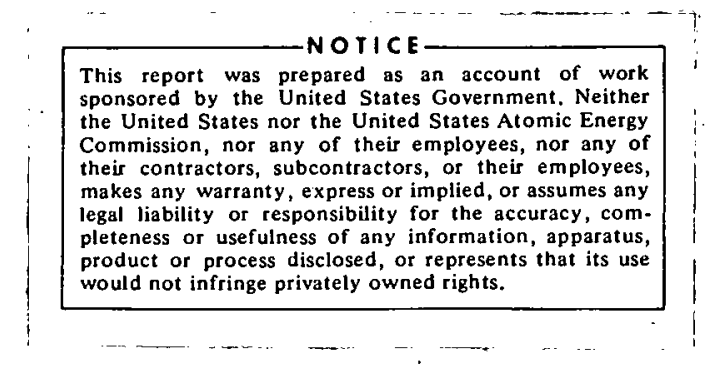

\section{COMMITTEE ON NUCLEAR SCIENCE}

NATIONAL ACADEMY OF SCIENCES - NATIONAL RESEARCH COUNCIL

\section{Washington, D.C.}




\section{DISCLAIMER}

This report was prepared as an account of work sponsored by an agency of the United States Government. Neither the United States Government nor any agency Thereof, nor any of their employees, makes any warranty, express or implied, or assumes any legal liability or responsibility for the accuracy, completeness, or usefulness of any information, apparatus, product, or process disclosed, or represents that its use would not infringe privately owned rights. Reference herein to any specific commercial product, process, or service by trade name, trademark, manufacturer, or otherwise does not necessarily constitute or imply its endorsement, recommendation, or favoring by the United States Government or any agency thereof. The views and opinions of authors expressed herein do not necessarily state or reflect those of the United States Government or any agency thereof. 


\section{DISCLAIMER}

Portions of this document may be illegible in electronic image products. Images are produced from the best available original document. 


\section{NOTICE}

The project which is the subject of this report was approved by the Governing Board of the National Re search Council, acting in behalf of the National Academy of Sciences. Such approval reflects the Board's judgment that the project is of national importance and appropriate with respect to both the purposes and resources of the National Research Council.

The members of the panel selected to undertake this project and prepare this report were chosen for recognized scholarly competence and with due consideration for the balance of disciplines appropriate to the project. Responsibility for the detailed aspects of this report rests with that panel.

Each report issuing from a study committee of the National Research Council is reviewed by an independent group of qualified individuals according to procedures established and monitored by the Report Review Committee of the National Academy of Sciences . Distribution of the report is approved, by the President of the Academy, upon satisfactory completion of the review process.

AEC Report No. C00-3368-4 
Herman Feshbach, Chaixsman.

E. Robert Beringer

Henry G. BTosser

Victor P. Bond

Walter L. Brown

John R. Huizenga

M. Stanley Livingston

Roy Middleton

Patrick Richard

Thomas A. Tombre110

D. Allan Bromley, Ex-Officio

Hans A. Bethe, Consultant

George L. Rogosa, Liaison

George W. Wheeler, Liaison

William S. Rodney, Liaison
Massachusetts Institute of Technology:

Yale University.

Michigan State University

Brookhaven National Laboratory

Bel2 Laboratories

University of Rochester

Santa Fe, New Mexico

University of Pennsyzvania

Kansas State University

California Institute of Tiechnology.

Yale University

Comezz University

Atomic Energy Commission

Atomic Energy Commission

National Science Foundation

\section{Expert Witnesses}

Charles M. Jones

John A. Martin

R. J. Silva

Paul H. Stelson

Alexander Zucker

Oak Ridge National Laboratory

Henry Motz

J. R. Nix

R. H. Stokes

Los AZomos Scientific Laboratory

Chell is Chasman

Harvey E. Wegner

Joseph Weneser

Brookhaven National Laboratory 
Expert Witnesses (continued)

Geoffrey F. Chew

Richard Diamond

Bernard Harvey

Earl Hyde

L. Jackson Laslett

Robert M. Main

Herbert Steiner

Lawrence Berkeley Laboratory

Lowell M. Bollinger

John P. Schiffer

Anthony Taylor

John P. Unik

Argonne National Laboratory

Robert E. Pollock

Indiana University

Lee Grodzins

Brookhaven Tandem Users Executive Committee

Harald H. Rossi

Columbia University

Herman D. Suit

Massachusetts General Hospital

Cornelius A. Tobias

Donner Laboratory

Fay Ajzenberg-Selove

Bernard L. Cohen

David A. Lind

Donald Robson

American Physical Society, Nuclear Division

J. M. Miller

American Chemical Society, Nuclear Science and Technology

Visitors

Herbert L. Kinney

John M. Teem

$\underline{N R C \cdot S \cdot t a f f}$

Paul W. McDaniel, Executive Secretary (until Sept. 30, 1973:)

Charles K. Reed

Janet M. Nunn 


\section{PREFACE}

In response to a request of the U.S. Atomic Energy Commission, the President of the National Academy of Sciences appointed an Ad Hoc Panel under the NAS-NRC Committee on Nuclear Science to make an intensive study of various aspects of the science and technology involved in heavy ion research in light of the needs of the national program in this field. (Appendix 1 contains the AEC letter to Dr. Handler, dated June 12, 1973.) In particular, the Panel was asked to formulate and evaluate various options for the development of heavy ion facilities that would include an appropriate accelerator complex capable of producing heavy ion beams and the ancillary apparatus required for experimental exploitation. These options have differing capabilities as objectives. The Panel was not asked to choose among these various options or among the various specific proposals that have been made to the Atomic Energy Commission. In addition, the Panel was asked to evaluate the current status and potentialities of the SuperHILAC and Bevalac accelerators of the Lawrence Radiation Laboratory of the University of California, Berkeley. The Panel was not asked to consider the overall scientific motivation for, or the sitings of, facilities.

The Panel consisted of a number of nuclear physicists and chemists, some of whom have broad experience in accelerator design and construction, an accelerator physicist, and representatives of various fields (biology, medicine, material science, and atomic physics) in which heavy ion beams would be an important research tool. A consultant provided an evaluation of the Bevalac research program for the Panel.

The major meeting of the Panel occurred July 15-17, 1973, at the National Academy of Sciences. Presentations were made by representatives of (1) the scientific societies (the Division of Nuclear Physics of the American Physical Society and the Division of Nuclear Science and Technology of the American Chemical Society) concerned with heavy ion nuclear research; (2) institutions that have been actively considering the development of major heavy ion facilities, including the Argonne National Laboratory, the Oak Ridge National Laboratory, and to a lesser extent the University of Indiana; (3) the Lawrence Radiation Laboratory at Berkeley, who reported on the SuperHILAC, the Bevalac, and the Electron Ring Accelerator; (4) research scientists involved in using heavy ion research facilities. In addition, specialists in the application of heavy ions to biology, medicine, and the material sciences testified. 
The Panel also considered a proposal by Milton $G$. White for the recommissioning of the Princeton-Pennsylvania Accelerator as a national heavy ion facility. The Panel is grateful to all these scientists for their valuable insights and assistance.

The report that follows opens with a summary of the findings of the Panel and continues with a brief resume of the motivation for heavy ion research, delineating the opportunities that would present themselves for nuclear studies and for application to the fields of atomic science, space, and astrophysics, material science, as well as biology and medicine. In view of the charge to the Panel, the main function of this discussion is to set the stage for developing the design criteria for the accelerator and ancillary apparatus that the national heavy ion program requires. It is not intended to provide an exhaustive justification for such a program, but it is hoped that the material presented will make possible appreciation of the significance of the forefront research that could be realized with advanced heavy ion capabilities.

Following a description of heavy ion facilities, both in the United States and abroad, the characteristics of the heavy ion accelerator needed for various aspects of the national program in heavy ion physics are outlined. The various technical alternatives available for the accelerator complex, their scientific and technological capabilities, and their costs are discussed. The ancillary facilities required for a national heavy ion accelerator facility and their costs are discussed as are manpower needs. This review concludes with a description of additional needed research and development of accelerator concepts and structures. 
I. FINDINGS 1

II. MOTIVATION 6

A. Introduction 6

B. Heavy Ions in Nuclear Physics and Chemistry 7

1. Macroscopic Properties 8

2. Microscopic Properties 10

3. Electromagnetic and Weak Interactions 13

4. Relativistic Heavy Ions 13

$\begin{array}{ll}\text { C. Atomic Physics } & 15\end{array}$

D. Heavy Ions and Astro and Space Physics 16

E. Material Science and Solid State 17

1. Reactor Damage Simulation 17

2. Materials Property Changes by Ion 18

3. Fundamental Solid State Research 18

F. Biomedicine 19

III. EXISTING HEAVY ION FACILITIES 22

A. United States Facilities 22

1. Electrostatic Accelerators 22

2. Cyclotrons 24

3. RF Linear Accelerator ... 24

4. Synchrotron 26

B. Heavy Ion Accelerators Outside the U. S. 28

1. UNILAC (West Germany) 28

2. ALICE and GANIL (France) 31

3. Dubna (Soviet Union) 32

4. The DARESBURY Accelerator (Great Britain) 32

5. Chalk River (Canada) 33

$\begin{array}{ll}\text { 6. Israel } & 33\end{array}$

IV. ACCELERATOR AND ANCILLARY EQUIPMENT CHARACTERISTICS 34

A. Characteristics for Physics and Chemistry Research 34

1. Resolution 35

2. Variability 38

3. Angular Resolution $\quad 40$ 
B. Characteristics for Biomedical Research 40 .

C. Characteristics for Atomic Physics 42

D. Characteristics for Materials Science and 42 Solid State Physics

E. Ancillary Facilities $\quad 42$

v. TECHNICAL ALTERNATIVES AND COSTS $: 46$

A. Introduction 46

B. Large Tandem Accelerator 48

C. Tandem-Cyclotron Systems 49

1. Magnet 50

2, Radiofrequency Systems 52

3. Injection 54

4. Extraction $\quad 55$

5. Beam Quality 55

D. Cyclotron - Cyclotron Systems $\quad 58$

E. Linear Accelerators b 61

1. Superconducting vs. Room Temperature 61

$\begin{array}{ll}\text { Structures } & 61\end{array}$

3. Accelerating Structures 62

4. Focusing 63

5. Beam Injection 63

6. Summary 64

F. Upgrading of Existing Facilities 64

1. SuperHILAC 66

2. Bevalac 67

3. PPA 67

G. User Needs, Manpower Considerations and 67 Operating Costs

H. Costs 72

I. Additional Research and Development 75

1. Ion Source $\quad 75$

2. Beam Pulsing 76

3. Multiple Beams $\quad 76$

4. ERA Concept 76

$\begin{array}{ll}\text { APPENDIX } & 77\end{array}$ 


\section{FINDINGS}

The use of accelerated nuclei of elements heavier than helium presents scientists and engineers with unique opportunities to make significant advances in the understanding of nuclei and nuclear interactions, atoms, solids, the structure of materials, and the properties of living tissue. This understanding offers important opportunities for application to both basic and applied fields, such as astrophysics, medicine, and nuclear reactor technology. These opportunities are being increasingly exploited within the scientific community.

In consideration of facilities offering differing levels of capability in heavy ion science, three options were considered.

OPTION: Take full advantage of the opportunities in heavy ion science and maintain the momentum of the present national effort. The investment in funds and manpower would be commensurate with the effort being made by other countries. It would involve the establishment of a national facility which would provide beams of ions as massive as uranium, with energies of the order of $10 \mathrm{MeV} /$ nucleon and with intensities of the order of $10^{12}-10^{13}$ particles/sec.* Beam characteristics and detection capabilities would permit the performance of precision experiments. The capability of this facility would be complementary to that of the SuperHIIAC.

OPTION: A substantially reduced national capability. In comparison to the first option the range of experimental parameters which the heavy ion system would make accessible to investigation would be considerably restricted; important classes of phenomena envisaged in the first option would be inaccessible. Option II would provide facilities which would produce beams of heavy ions with atomic number whose maximum would be in the middle of the periodic table. These facilities could be so designed as to permit later expansion to the system of greater capability envisaged in Option I.

OPTION: Extend the present capability for research with heavy ions by upgrading and/or extending facilities now operational. This option could be implemented concurrently with either Option I or Option II.

* One particle per second = one heavy ion per second. 
The implementation of these options was evaluated in terms of technical feasibility and scientific need. The findings of the Panel are as follows:

$\checkmark \quad$ 1. A system consisting of a large electrostatic accelerator injecting into an isochronous cyclotron is technically feasible and satisfies the scientific requirements of Option I. Its cost and construction time can be estimated realistically. For these reasons this facility is recommended by the Panel if Option I is to be implemented in the near future. A special advantage of this system (and of the two cyclotron system (GANIL) proposed by the French) is.its ability, for a given design energy for the heaviest ion species, to provide extremely energetic beams of the lighter heavy ions, opening up an area of investigation not accessible, for example, to the West German linear accelerator, the UNILAC. Among other things, this capability can be of great importance for biomedical applications. It is the opinion of the Panel that Option I is essential if the U.S. activity in heavy ion science is to remain competitive.

In arriving at this finding the Panel considered other systems which might involve superconducting cyclotrons, superconducting and room temperature linear accelerators with helical and spiral resonator cavities, and other accelerator technologies such as those based on the electron ring accelerator concept. These were judged by the Panel to require further research and development before a realistic judgment of the performance and costs of such a system can be made. In view of their great potential the Panel recommends that such research and development be vigorously pursued. This recommendation applies with equal force to Option II and Option III.

$\checkmark \quad 2$. The Panel was impressed by the capability for heavy ion research offered by electrostatic tandem accelerators with terminal voltage of the order of $20 \mathrm{MV}$. These could be used independently or as injectors into existing cyclotrons, although it should be emphasized that none of the latter have been designed specifically for such use. Consequently the capabilities of such systems are correspondingly limited. Such electrostatic accelerators are being offered commercially. Heavy ions up to the middle of the periodic table could be accelerated by such systems with ample currents for precision experiments. Facilities could be so designed as to permit later expansion to a system of greater capability. However, if the U.S. is to remain as a leader in the field of heavy ion science, this possibility of expansion of heavy ion science to Option I must be implemented in the near future since Option I facilities abroad will soon become operational (UNILAC in 1975, GANIL possibly in 1980). 
$\checkmark \quad 3$. The Panel strongly recommends an improvement program for the SuperHILAC with emphasis on the redesign of the high voltage injector for the heavier ions so that reliable performance can be assured. The SuperHILAC is potentially a most important element of the national heavy ion program; it is essential that this potential be realized. The Panel assígns a high priority, not only to the improvement of the SuperHILAC, but also to the implementation of an effective program for outside users of it. The SuperHILAC will provide neither medium and light heavy ions as energetic as those envisaged in Option I, nor the beam energy resolution and the simultaneous precise beam energy variability. In terms of the national effort this situation would be partially ameliorated for only the lighter heavy ions (below argon) by beams which could be produced by a $25 \mathrm{MV}$ electrostatic accelerator.

Upgrading of other operating accelerators will, for the most part, permit important extensions of programs now in progress. However a qualitative change in the experimental parameters, such as energy and ion species is possible only with Option I, and to a lesser extent with Option II .

$\checkmark \quad$ 4. The Panel was asked to comment on the proposed program for the Bevalac facility which is now under construction. The Bevalac takes advantage of the unique opportunity presented by the geographical propinquity of the SuperHILAC and the Bevatron. However, the operational costs are relatively very large. Relativistic heavy ions are uniquely important for the study of the passage of heavy ions through living tissue with the possible ultimate goal of the use of heavy ions for cancer therapy. The Panel recommends that the needs of biomedical scientists should govern principally the rate and the scale of Bevalac development. In the opinion of the Panel, at the present time, it has not been demonstrated directly, or implied by proposal pressure of outside user physicists and chemists, that the probability of finding important new nuclear phenomena unique to relativistic heavy ions is sufficiently high to provide primary justification for the operation of the Bevalac.

The Panel did not recommend recommissioning the Princeton-Penn accelerator, for it appears that the Bevalac offers sufficient capability for the anticipated needs of relativistic heavy ion science.

$\checkmark \quad 5$. Without providing extensive ancillary equipment for detection, for on-line data acquisition and data reduction, for hot target handling, etc., it will not be possible to realize the experimental opportunities offered by the proposed new heavy ion accelerators. The Panel recommends that the national program for heavy ions include adequate provision for such equipment. 
$\checkmark \quad 6$. The range of the nuclear species and the physical characteristics of the beams provided by heavy ion accelerators are especially sensitive to the properties of the ion sources. It is vitally important that ion sources capable of producing intense beams of ions throughout the periodic table be made available, that the sources have a sufficiently long lifetime and that they can be readily interchanged. Continued research and development of ion sources could yield important benefits; the Panel strongly recommends a coherent national program in this area. The performance of a radiofrequency accelerator as a high resolution research tool depends on efficient "beam pulsing" -- that is, on the efficient injection of short, intense beam bursts into the se structures . Both the circular and linear radiofrequency accelerators have special problems in this regard. It is the recommendation of the Panel that the unfortunate lag in research and development in this area be corrected immediately.

Formal presentations regarding the national heavy ion program were solicited from representatives of the Division of Nuclear Chemistry and Technology of the American Chemical Society and of the Division of Nuclear Physics of the American Physical Society. The latter solicited letters from their fellow members, which were presented to the Panel.

For the most part, there was a genuine enthusiasm for heavy ion physics in the letters received by the Division of Nuclear Physics from its members . However, some of them expressed concern that the traditional methods of nuclear research would be affected adversely and some corresponding values would be lost if large national facilities were to be funded. Some expressed the fear that the number of nuclear physicists receiving federal support would be reduced. The testimony of the society representatives, together with the input from the aforementioned letters form, in part, a basis for the following recommendations.

The Panel strongly recommends that facilities constructed under a national heavy ion program, including the SuperHILAC, be national facilities . It is critically important that the se facilities be available to all scientists on a priority basis determined by scientific merit.

It is also essential, and we cannot emphasize this point too strongly, that sufficient funds be programmed for effective outside user participation, and that sufficient resources at the facility in terms of equipment, support personnel, and housing be available to meet user needs. The management of these facilities should be especially sensitive to the needs of the outside users. 
Construction of heavy ion facilities which would maximize the number of users should be encouraged. This recommendation gives particular weight to systems which at a given time provide more than one independent beam for research.

Special consideration should also be given to "in-house" users to ensure sufficient local strength for improvement and maintenance of the accelerator. for the design, construction, and maintenance of ancillary equipment, and for provision of assistance to outside users in the planning and execution of experiments to be performed at the facility. 


\section{MOTIVATION}

\section{A. Introduction}

The phrase "heavy ions" in the context of this report refers to ionized atoms more massive than helium. The accelerators which will be discussed are to be capable of producing beams of the se ions with energies which, according to the proposed accelerator designs, could cover some portion of a range extending from several $\mathrm{MeV} /$ nucleon through several hundred $\mathrm{MeV} /$ nucleon to as much as several $\mathrm{GeV} /$ nucleon. The acceleration of a wide variety of atomic species up to and including uranium has been considered.

Heavy ion beams, like beams of other particles, can be used to probe the properties of matter. These beams,upon striking targets composed of various materials,produce a broad range of phenomena which advance understanding of the structure of nuclei, of atoms, of solids and of the nature of the electromagnetic field. This area of endeavor was selected by the Physics Survey Committee of the National Academy of Sciences as one of fifteen, from a total of some seventy research areas covering all subfields of physics, whose "growth potential warrants high priority for support".* As is the case for any frontier field, research with heavy ions can be at the boundary of our understanding, penetrating into the unknown with tools possessing very special and specific attributes which we shall briefly describe below. It may be expected that discoveries in a frontier field will require the development of new insights as well as the creation of new concepts, some of which will undoubtedly prove to be valuable in other fields. This last expectation is based on the historical fact that differing forms of matter will, under suitable conditions, exhibit analogous behavior requiring a quite similar conceptual basis for their understanding.

The discoveries which are made and the properties of matter which are uncovered can have immediate important consequences for other scientific areas as diverse as material science and astrophysics. They will have application to industrial and societal problems such as those involved in energy generation. Investigations with biological materials may provide new understanding of, and perhaps even new techniques for, the treatment of cancer.

The successful realization of these opportunities requires engineering and scientific capabilities of high order. Sophisticated apparatus including accelerators and detection and analysis equipment will need to be designed and constructed. Computers will play an important role in all aspects. To some extent, all these developments push forward the frontiers of known technology; new skills and advances in technology will be needed. These, as past experience has demonstrated, can have a wide range of scientific and engineering applicability.

* Physics Survey Committee, Physics In Perspective. Washington, D.C.: National Academy of Sciences, 1972. 
The special properties of heavy ions which make them so useful are their high positive charge, their large mass, and their ability to permit the transfer of a large number of nucleons from one nucleus to another.

Because of their large charge, they can be accelerated to very high energies once a sufficiently large fraction of their atomic electrons have been removed. As a consequence of its large charge, a heavy ion is a source of strong electric field making possible the study of matter in the presence of very strong electric forces.

Because of its large mass the heavy ion has a very large momentum, much greater than, say,a proton of the same energy. In another comparison a $16.8 \mathrm{GeV}$ electron has the same momentum as the heavy tin ion, ${ }^{120} \mathrm{Sn}$, with an energy of $10 \mathrm{MeV} /$ nucleon. Because of this large momentum, a heavy ion in collision with a nucleus can propel that nucleus forward with a high velocity. If the collision is a glancing one, either or both nuclei can be set into very rapid rotation. Because of their high momenta, heavy ions have a very short wavelength. A $120 \mathrm{Sn}$ heavy ion with $10 \mathrm{MeV} /$ nucleon has a wavelength/2 $/$ of about $1.3 \times 10^{14} \mathrm{~cm}$. A heavy ion can thus act like a short wavelength microscope exploring properties of nuclei which involve these very short dimensions. This microscope effect differs in character from that of an electron of a similar wavelength because of the large size of the colliding systems and their composite nature, with the consequence that this property of heavy ions can be exploited principally near the surfaces of the colliding nuclei.

Since the heavy ion has a composite character, it can transfer clusters of nucleons to, or accept such clusters from, other nuclei,forming in this way entirely new nuclear species. Reactions occur in which the colliding nuclei fuse to form a compound system which then fissions into two or more fragments . At sufficiently high energies, a collision between nuclei (except possibly for those which are glancing) will almost certainly give rise to a nuclear reaction. From the point of view of the incident heavy ion, the central region of the nucleus with which it collides is completely absorbing.

These are the gross properties of heavy ions which can be exploited in the studies of nuclei, atoms, and the structure of materials. More subtle and equally important characteristics of heavy ions will become evident in the course of the description which follows of the use of the se ions in each of these areas.

\section{B. Heavy Ions in Nuclear Physics and Chemistry}

The study of the collision of heavy ions with nuclei will severely test our understanding of nuclei and of nuclear reactions. The new phenomena which will be discovered will be interesting not only in themselves but also because 
they will undoubtedly require a revision and reformulation of our concepts as well as the introduction of entirely new points of view. The ways in which heavy ion beams can be exploited and the nature of the nuclear properties which can be probed are briefly summarized below. The latter are conveniently grouped as macroscopic properties and quantal or microscopic properties. This will be followed by a discussion of important opportunities, unique to heavy ion beams, for the measurement of electromagnetic and weak interaction properties of nuclear levels. The final section will be devoted to experiments with relativistic ions, with particular reference to the Bevalac.

\section{Macroscopic Properties}

In a heavy ion collision, measurements may be interpreted in terms of parameters describing the target nucleus, the incident heavy ion and on occasion the compound nucleus. One can ask for such bulk properties of nuclear matter as compressibility and viscosity and their dependence on excitation energy. Stated more generally, one can ask for the dependence of the energy of a system of $A$ nucleons on such macroscopic parameters as shape, density and charge. Such a system can also exist in fragments, and the dependence of the energy on the number of fragments and the shape, density and charge of each,as well as their relative separation,are clearly of primary importance for the understanding of the reaction mechanisms involved in heavy ion interactions and fission. The inertial parameters such as effective mass and moment of inertia scale the accelerations of the macroscopic parameters of the system induced by external forces and are therefore needed in order to describe the dynamics of the nuclear system. It is to be emphasized that present day information on the se properties, both experimental and conceptual, is spotty and rudimentary at best . Obviously such insights and data are needed for the empirical understanding of fusion, of fission, and of the formation of new elements, to mention only three of many important examples. It is also important to realize that these properties will be revealed only following the systematic study of the collisions of a variety of nuclei at a variety of energies. It is necessary to investigate reactions involving heavy nuclei colliding with light and heavy nuclei, spherical nuclei colliding with other spherical nuclei, deformed nuclei colliding with spherical ones or deformed ones, vibrators colliding with rotators, and so on.

The macroscopic properties of the surface of nuclei can be investigated by heavy ion collisions. Multiparticle transfers--that is reactions in which a number of nucleons are transferred from one nucleus to the other--are sensitive to these properties. Recently the concept of friction was introduced as an element in the macroscopic description of the interaction occurring at the surfaces of two colliding nuclei. It is thought to play an important role in the fusion process. Interference phenomena at small angles (recall the small values of wavelength $/ 2 \pi$ ) directly probe the interaction in the surface regions. The 
measurements necessary to delineate these phenomena are for the most part yet to be done. Observation of the interference phenomena requires an excellent angular resolution which needs to improve as the masses of the nuclei involved increase.

The formation of new nuclei, mentioned earlier, is one of the most important goals of the heavy ion program. Production of new nuclei may occur in several ways. It may involve a multiparticle transfer. It may involve the amalgamation of the target and projectile nuclei to form a compound system which then fissions. A third mechanism involves intimate contact between two nuclei, which is, however, not of sufficient duration so that a true compound system is formed. This process referred to as the fusion-fission process is an important reaction channel which is not understood at the present time. The circumstances under which it competes with or dominates the other two processes are not yet known. There are also considerable uncertainties in the quantitative description of the particle transfer and the compound nuclear process. A fourth process, which is most effective when the projectile has very high energy, is fragmentation. As a consequence of the impact, both projectile and target break up into fragments, some of which are new nuclei.

Regardless of the mechanism, the properties of new nuclear species which are formed will provide new data from which a new understanding of the ground states of nuclei can be developed. It has been emphasized often that our understanding of the systematics of these states, upon which a great deal of experimental and theoretical effort has been expended because of their fundamental importance, is based on the nuclei in the stable valley. This refers to the surface which results when the energy of these nuclei is plotted as a function of the number of neutrons (N) and number of protons(Z). Since the se nuclei are stable, they have the lowest energies at special values of $\mathrm{N}$ and $\mathrm{Z}$, the locus of which forms the bottom of the valley. These are just a fraction $(\sim 5 \%)$ of the nuclei which will not decay by the emission of neutrons or protons or by heavy ion collisions. Will the understanding of the nuclei based upon the properties of the relatively few stable nuclei plus our knowledge of underlying nuclear forces suffice for the new 95\%? If it does not, it may well become necessary to revise our understanding of nuclear structure, its relation to nuclear forces and/or the nuclear forces themselves. It is probably not necessary to emphasize the fundamental nature of these issues which impinge 
directly on one of the principal goals of all of physics -- the discovery and delineation of the properties of matter.

The possible existence of superheavy nuclei epitomizes, in an extreme way, the problems raised in the above discussion. It is surmised,by extrapolation of present information based on the stable valley, that there will be an island of stability* in the neighborhood of $Z=114$. This is a considerable extrapolation and, indeed, there is not complete agreement on the best extrapolation procedure. But the existence or non-existence of these elements and their lifetimes will be a sensitive test of the concepts underlying our present description of nuclei. In addition, there is the question of how these nuclei, if they are stable, can best be created. Involved in this question are all the macroscopic properties of nuclei mentioned above. The viscosity of nuclear matter, the energy surface, the dynamics, the role of friction, the deformability of the interacting nuclei, and the effect of the very strong electrostatic fields which exist between the final products of the reaction all play important roles.

\section{Microscopic Properties}

The same encounters which provide opportunities for the study of the macroscopic properties of nuclei also permit the investigation of the quantum states of these complex nuclear systems. The se experiments require high quality incident projectile beams as well as high resolution detection systems. An outstanding problem of modern physics is the quantum nature of matter, and heavy ion physics can undoubtedly play a seminal role in dealing with this problem. Different forms of matter exhibit under appropriate circumstances similar modes of motion. The superconducting state is found in both metals

* Stability in this case refers to stability against fission. These nuclei will generally be unstable against alpha and beta decay. Lifetimes as long as several thousand years are predicted in some extrapolations, however. 
and nuclei, rotational states in both molecules and nuclei, vibrational states in solids, molecules and nuclei, the shell model in atoms and nuclei. The realization that these quite similar degrees of freedom can occur even under a wide variety of physical circumstances has been extraordinarily fruitful and challenging. As a consequence the impact of discoveries in various fields of physics on nuclear physics and vice versa has been remarkable, demonstrating the unity of physics forged by common concepts and phenomena.

Heavy ion physics is expected to contribute to this program in two ways. First, new modes of nuclear motion may well be discovered. Major progress in nuclear physics has been made in the past with the discovery of the dominance of special degrees of freedom for particular ranges of nuclear species and experimental parameters. The shell and rotational models are examples. Heavy ion physics expanding as it does, the experimental circumstances under which a much greater variety of nuclei can be studied may provide further examples, some of which are suspected now, some of which may be completely surprising. These nuclear modes of motion are generally approximate; their deviations from an exact description of nuclear states which is manifested by the coupling between the various degrees of freedom provide clues to the underlying reasons for their overall validity. Heavy ion physics, because it provides more extreme conditions, displays these couplings among nuclear modes in an unmistakeable and unambiguous fashion. An example of the usefulness of these investigations has already been developed in the collision of the lighter heavy ions with deformed nuclei where, inter alia, the coupling between rotational and vibrational degrees of freedom plays an important role.

Production of states which are either new members of known families or examples of entirely new types is possible because of the special properties of heavy ion beams. Because of their high linear momentum they can in a grazing collision set the target nucleus and themselves into rapid rotation thus producing states with large values of angular momentum. Because of their intense Coulomb fields it is possible for them to excite target nuclei by multiple Coulomb excitation, thus generating states which would appear weakly if at all in collisions with, say, electrons or protons. Perhaps the most fertile source of new states will be in transfer reactions in which the target nucleus accepts from, or transfers to, the projectile clusters of nucleons. Transfer of clusters containing as many as twelve particles have been observed. When such transfers are especially favored (after kinematic effects have been factored out) the states which are formed are particularly interesting since they may form families of states with common attributes which the reaction selectively displays. Since high excitation energies are possible, some of the states that are found may be energetically particle unstable. However, if they are readily observed their decay by particle emission must be inhibited. These are then 
very special states distinguished by their unusual stability. In either case, there is the distinct possibility that these states are manifestations of new degrees of freedom of the nucleus with a resultant grouping of states into families and with associated selection rules which ensure their stability. These selection rules and degrees of freedom will become especially interesting if they can be related to symmetry principles.

The history of nuclear physics provides several examples of this kind of development. Recent cases include(l) isobaric analogs which are directly related to the isospin degree of freedom and the charge independence of nuclear forces and, (2) isomeric fission, a phenomenon associated with ranges of greater stability which appear as the nucleus becomes more deformed.

Heavy ion physics and particularly transfer reactions are significant for this important type of research since they increase enormously the variety of nuclei, and the range of excitation energy and angular momentum, which can be investigated. Fortunately, this is a controlled flexibility in the sense that, by appropriate choice of a projectile and its energy, a particular range of excitation energies and angular momenta of the target nucleus referred to as "windows" can be preferentially selected for examination. It is this combination of flexibility and specificity which is a consequence of the large size, composite character, and large charge which makes the heavy ion unique as a tool for studying the nucleus.

Another method which has been used for the discovery of new kinds of states has been the observation of anomalies in the elastic and inelastic scattering of nuclei. In the case of lighter nuclei ${ }^{12} \mathrm{C}+{ }^{12} \mathrm{C},{ }^{12} \mathrm{C}+{ }^{16} \mathrm{O},{ }^{16} \mathrm{O}+{ }^{16} \mathrm{O}$, the dependence on energy of the probability for elastic scattering through a given angle has shown a remarkable structure, with maxima and deep minima, which has persisted to relatively high energies. One of the interpretations of this data has suggested that the two nuclei form a relatively loose molecular type system. Such structure in elastic scattering is expected to diminish with increasing mass of the interacting nuclei but will presumably still make its appearance in appropriately chosen reactions.

Recently heavy ion nuclear reaction studies have discovered remarkably sharp states high in the nuclear continuum. These states in the $35-40 \mathrm{MeV}$ range in ${ }^{24} \mathrm{Mg}$ and ${ }^{28} \mathrm{Si}$. for example, were totally unexpected. Even more striking is recent evidence that suggests the persistence of these structures in adjacent nuclei having additional valence nucleons which broaden the width of the state but otherwise perturb the core configuration very little. It must be emphasized that these states are unobservable without an accelerator which produces beams having simultaneously excellent beam energy resolution 
and rapid, convenient and precise energy variability, so that the resonances corresponding to the new sharp states can be delineated and studied.

These new states may well form an entirely new nuclear spectroscopy -- both experimental and theoretical. It should be noted that their study rests entirely on the availability of high quality heavy ion beams; it should also be noted that as high quality beam energies are increased, experience thus far has been that totally new phenomena, unobservable with poorer quality beams, continue to emerge. There is every reason to expect that this will continue to characterize heavy ion science as it moves into new energy regimes with precision.

\section{Electromagnetic and Weak Interactions}

With heavy ion beams, it becomes possible to measure a number of the electromagnetic and weak interaction properties of nuclear levels. Many of these methods are a consequence of the large linear momentum of these projectiles. For example, the spin of nuclei in high spin states that are produced by grazing collisions will be preferentially oriented perpendicular to the plane of scattering. The radiation produced as these high spin states decay will be anisotropic and it becomes possible to determine the multipole order of the radiation, its parity, and the associated transition rates. Because the target nucleus can be propelled out of the target by the collision, it is often possible to measure the lifetime and the magnetic moments of the excited nuclear states produced in the collision. The same recoil effect is exploited in a new recoil mass spectrometer which should be an important tool for determining the nature of the reaction products. Because of the strong Coulomb field associated with the heavy ion, it will be possible to determine the quadrupole moments of excited nuclear states. Because the exotic nuclei far from the stability valley which will be formed in heavy ion collisions are unstable against $\beta$-decay, it will be possible to populate a large number of levels in the daughter nuclei and thereby obtain important new nuclear information as well as new insights into the nuclear weak interaction. It is not possible in this brief listing to provide an understanding of these measurements; it is, however, clear that heavy ion physics will make possible the measurement of a large variety of properties of excited states of nuclei which for the most part cannot be obtained by other methods. There is a very high probability that these data will play a very essential role in the revision in our understanding of nuclei which heavy ion physics will undoubtedly stimulate.

\section{Relativistic Heavy Ions}

The development of heavy ion beams with energies of 2.1 $\mathrm{GeV} /$ nucleon has opened an entirely new area for investigation. The almost complete lack of experience in this experimental domain makes it difficult to 
prophesy the impact that research with these beams will have. On the other hand, it equally follows that it is likely that some surprising results will be obtained. What remains in question is how important they will be. In the presentation that follows, the experimental opportunities which present themselves, as well as some indication as to the extent to which they are speculation, will be described. Experiments which can be done equally well with simple beams (protons, electrons, etc.) are omitted.

In the realm of macroscopic heavy ion physics, it is clear that $\mathrm{GeV} /$ nucleon collisions of heavy ions with nuclei will result in the production of many new nuclear species. The relative probability for the production of a given nucleus with relativistic heavy ions will differ of course, from that produced by other projectiles such as relativistic protons or lower energy heavy ions. The observation of these new nuclei may be less difficult with relativistic heavy ions because of their high linear momentum. Even if reaction products fragment, the fragments will, by and large, continue to move with the original velocity of the heavy ion and, therefore, can be readily detected in the forward cone. The process of fragmentation is itself of interest and some results in this regard have already been obtained.

At a more speculative level are the possible excitation of shock waves and the production of "holes" punched out of nuclear matter. It is thought that, in collisions at these energies, regions with nucleon and energy density substantially above normal values may be generated. It is, of course, most informative for our understanding of nuclear matter to see how it behaves under such extreme conditions. Appropriate methods for the detection of the se various phenomena, however, remain to be developed.

Because of the high energy per particle, it seems reasonable to expect a substantial production of kaons and associated hypernuclei. The se hypernuclei can themselves be studied, while the kaons might be used either as probes of nuclear structure by elastic or inelastic processes or to study hypernuclei by means of strangeness changing reactions in kaon-nuclear collision. More speculative in nature is the possible formation of multiple hypernuclei, that is, nuclei containing several hyperons in the collision of the relativistic ions with nuclei. Hypernuclear physics is another and very promising branch of physics. For further discussion, the reader is referred to a 1973 Brookhaven National Laboratory summer study.

Pions would also be generated in a heavy ion-nucleus collision. Here the interesting experimental parameter is the pion multiplicity when two nuclei collide as compared with multiplicity when the incident particle is a proton, and when two nucleons collide. 
The Bevalac could also produce high energy deuterons which in turn could be stripped to provide high energy neutron beams. These would be useful in many nuclear investigations. It is however not clear how these beams would compare with neutron beams produced by the collision of high energy protons with deuteron or beryllium.

There are important potential uses of the Bevalac heavy ions in biomedicine and in astro and atomic physics. These are discussed in a later section.

\section{Atomic Physics}

As heavy ions pass through matter, they will ionize and be ionized by the atoms with which they collide. They will acquire electrons from the atoms or they will lose electrons to the atoms, a process called charge change. They will also excite and be excited by the atoms and both atoms and ions will emit radiation and electrons in the course of de-excitation after collision. All of these processes contribute in an essential fashion to the effects of heavy ions on materials, as well as on living material. After passing through a foil or gas the ions will be in various charge states. This process of stripping is in fact an essential step in the production of high energy heavy ion beams, since it is much more advantageous to accelerate more highly ionized ions. An understanding of ion charge states should then enhance our ability to design more efficient, less expensive accelerators. These matters are discussed in later sections of this report; in this section we consider what can be learned about atomic structure, about the radiative and electron emission (Auger) processes and about the reaction mechanisms which operate when atoms collide.

With a high energy, heavy ion facility it will be possible to study the spectroscopy of highly stripped atoms. Remaining atomic electrons will be under the influence of a much stronger electric field and will be moving more rapidly than is usually the case for the outer and inner electrons of an atom. Both relativistic and quantum electrodynamic effects become important. Thus from a very fundamental point of view our understanding of two of the most important cornerstones of modern physics can be tested under a new set of circumstances where they are major effects rather than small perturbations.

The transient formation of combined atoms (in ion-atom collisions) whose net atomic number far exceeds $Z=92$ and whose lifetime during collisions is sufficiently long so that electronic radiative processes can occur in their presence, is now under study in many laboratories. For such large atomic numbers, rather unusual effects are predicted by quantum electrodynamics, providing thus a stringent test of its underlying concepts . 
Such studies will have important astrophysical applications. Highly ionized atoms are commonly present in stellar atmospheres. Knowledge of the typical radiations they emit and the absolute transition strengths will help to determine the properties of these atmospheres, and thus advance our understanding of stellar structure and energy production by stars.

The atomic reaction mechanisms involved in heavy ion collisions are at present poorly understood. Among the phenomena which require further investigation are the dependence of charge changing collisions of heavy ions in gases and dense media upon atomic species and energy. The excitation and ionization of inner electrons of the target atom seems to involve a number of different mechanisms, not all of which appear to be known and whose relative importance remains to be sorted out. Significant discrepancies between theory and experiment, in some cases involving several orders of magnitude, have been reported. These excitations are followed by $x$-ray emissions which may serve as a means of identification of the radiating atom. If the processes involved. are sufficiently well understood, this method might then become the method for the identification of new elements produced in heavy ion collisions.

\section{Heavy Ions and Astro and Space Physics}

Highly ionized ions occur naturally in stars and as a component of the cosmic rays (principally of galactic origin although a small fraction originates in solar flares). Heavy ions are also found in the earth's trapped radiation belt.

As previously noted, determination of the optical spectra of heavy ions and the associated transition probabilities will be uniquely useful in the interpretation of spectra of stars. It is worth remembering that stellar spectra form a most important, if not the most important, source of information on the internal constitution of stars and hence on their evolution and energy production (nucleosynthesis). Heavy ion reactions probably form the dominant nuclear reaction in explosive nucleosynthesis (e.g., as in supernova) which occurs in stars which have exhausted their supply of hydrogen and helium.

These explosions are probably responsible for the energetic heavy ions which are found throughout our galaxy. The energy spectrum of these elements has a broad maximum at a few hundred $\mathrm{MeV} /$ nucleon and decreases rapidly for greater energies. Lighter nuclei are most abundant; but from the point of biological effectiveness, the iron nuclei form the most important component. In interplanetary space one $A U$ from the sun, the flux of iron nuclei in an energy range from $100-1000 \mathrm{MeV} /$ nucleon is $0.5 \mathrm{nuclei} /\left(\mathrm{m}^{2} \mathrm{sec} \mathrm{sr}\right)$. The reactions these heavy ions can induce must be understood in order to reduce 
the biological hazards of manned space flights. Assessment of these hazards is presently difficult because of the lack of pertinent data.

Objects in space (e.g., meteorites) are also generally being bombarded by these heavy ions. The resultant reactions can be responsible for the presence of some elements in an abundance which differs from that found on the earth.

\section{E. Material Science and Solid State}

Materials science currently makes use of energetic heavy ions in three major areas; namely reactor damage simulation, ion implantation, and fundamental solid state research.

\section{Reactor Damage Simulation}

If the proposed use of breeder reactors and controlled thermonuclear fusion reactors for generation of power are to come to fruition, formidable material problems must be solved. The structural and cladding materials must preserve their integrity in the presence of neutron fluxes and operating temperatures that are much greater than those of present day reactors. The phenomena which have been observed include void development and accompanying material swelling, a high density of dislocations and drastic changes in bulk properties which are important for the operation of the reactor and the lifetime of its components. The experimental parameters which describe these phenomena have not been fully determined, nor has any complete theoretical description been developed.

It is not possible to duplicate the conditions which would be present in these advanced reactors using available neutron sources, and our understanding of radiation damage dynamics is so limited that it is not possible to predict the radiation damage which might occur. However, with heavy ion bombardment of the materials with self ions--that is, the material is bombarded with ions of the same atomic species which make up the material--it becomes possible to simulate the damage effects of neutrons. With just a few hours bombardment, it is possible to simulate the radiation effects which would accumulate over the course of a year inside a reactor.

More systematic investigations are required in order to establish the primary phenomena and the appropriate description of radiation damage to materials. In order to approach realistic conditions more closely, a wide variety of heavy ion species needs to be available and high energies are required. Energies up to, say, several hundreds of $\mathrm{MeV}$ for iron ions are 
required in order that thicker samples of material can be tested and bulk material behavior determined. Current designs of CTR reactors envisage the use of superconductors for the production of the magnetic fields required for plasma containment. The effect of intense neutron fluxes on superconducting properties can again be studied through simulation by heavy ion bombardment.

\section{Materials Property Changes by Ion Implantation and Ion Bombardment}

This area of materials science is growing rapidly, particularly in the semiconductor device field where the distances of ion penetration at a few hundred $\mathrm{keV}\left(10^{-5}-10^{-4} \mathrm{~cm}\right)$ match quite well the dimensions of currently interesting device structures and where the ion flux required for effective alteration of the electronic properties of semiconductors is relatively low and consistent with efficient manufacturing. There are specialized interests in deeper implantations, for example for certain buried optical wave guide structures, but still only in the range $10^{-4}-10^{-3} \mathrm{~cm}$, and hence accessible with beams from existing ion accelerators.

The prospect for useful material doping with very high energy heavy ions seems remote because of the massive ion fluxes that would be required to make any substantial compositional change in a thick layer deep in a solid. It is possible, however, that the large end-of-range damage effects produced by such ions might be found to have useful applications. It would seem prudent to recognize this as a potential area of materials interest.

Heavy ion track production in solids is an area which is already being actively studied. Heavier and more energetic ions would greatly facilitate this area of materials research, which has impact on areas from astrophysics (through rock dating) to the practical formation of micropore filters .

\section{Fundamental Solid State Research}

Magnetic fields and electric field gradients at impurities in solids can be measured through interactions with the magnetic dipole moment and electric quadrupole moment of nuclei which are excited and recoil when implanted into solids by heavy ion bombardment. Heavy ions can also be used to probe the constitution of materials, the lattice arrangement of various components of a crystal, as well as the ion force fields present inside a crystal. The phenomenon of channeling (the guiding of ion trajectories in a crystalline solid by correlated small angle collisions with ordered arrays of atoms) is important in much of this work. Channeling provides a tool for probing the interatomic potential in a crystal lattice. It allows investigation 
of the dependence of inelastic energy loss of ions on the location of ion trajectories with respect to the rows and planes of ratoms of a solid. Channeling is also sensitive to crystalline imperfections and impurities and provides a means of measuring certain types of departure from short and long range order. In all of these areas highly energetic heavy ions will extend the accessible range of investigation.

\section{F. Biomedicine}

Biomedical interest in accelerated heavy ions lies in basic radiobiology, in the evaluation of possible hazards of space flights, and in radiotherapy. The key ion property of interest is the very high value of linear energy transfer, or LET (numerically equal approximately to $\mathrm{dE} / \mathrm{dx}$ ). Radiation quality, freq uently characterized in terms of LET, represents an important variable in the study of mechanisms of radiation damage to tissue. Thus it is important to be able to control this variable accurately over a wide range of values of LET. Investigation of the effects of particles of different LET, using the techniques of radiation chemistry, biochemistry and radiobiology, is needed to aid in the understanding of mechanisms involved in producing biological effects.

Exposure of cell populations to high-IET radiations results in "single hit" inactivation kinetics. One also obtains, compared to $\mathrm{x}$ - or gamma radiation, a high relative biological effectiveness or RBE, i.e., a greater effect per unit of dose. With low-LET radiations, the degree of effect is dependent on oxygen tension, and hypoxic cells may be protected by a factor of as much as 2.5 to 3 (the oxygen enhancement ratio, or OER). This dependence of degree of effect on oxygen tension is markedly reduced with very high LET radiations (OER approaches unity).

The Yale HILAC has been used* to study the kinetics of cell inactivation, $\mathrm{RBE}$, and the oxygen dependence of the effects of stripped heavy ions of increasing $\mathrm{Z}$, up to $40_{\mathrm{A}}$. In these studies, the inactivation cross section was found to increase with increasing LET until, at values of LET obtainable with $20 \mathrm{Ne}$, the inactivation cross section equaled. approximately the measured cross section of the cell nucleus. Surprisingly, at ${ }^{40} \mathrm{~A}$ the inactivation cross section appeared to rise sharply to equal approximately the measured cross

* Todd and Skarsgard, et al., Todd, P., Heavy Ion Irradiation and Cultured Human Cells, Rad. Res. Suppl . \&:196-207, 1967.

Skarsgard, L. D., et al., Survival, Chromosome Abnormalities, and Recovery in Heavy-Ion- and x-irradiated Mammalian Cells, Rad. Res., Suppl. $7: 208-221,1967$. 
section of the entire cell. These findings may indicate a previously undocumented mechanism of cell inactivation and should be confirmed and extended, using improved experimental techniques that are now available. The basic mechanisms involved should also be studied.

Recent work in Berkeley (Kelly, L.) indicates that stripped carbon atoms accelerated to energies sufficient to penetrate the entire body of a mouse have a very high RBE for the induction of at least one kind of neoplasm. This observation (particularly if it holds for other ions and for other tumors) is of obvious importance not only with respect to possible hazards of extended flight, but in the potential use of heavy stripped ion beams for radiotherapy. This may represent an example of the importance of being able to expose large tissue volumes to radiations spanning a wide range of LET's, possible now only to a limited degree by means of fast neutrons. It is now possible to study malignant cell transformation in tis sue culture (Borek, C.; Columbia University and Brookhaven); thus some studies of this nature could be done with heavy ions of relatively low energy.

Interest in stripped heavy ions for radiotherapy stems largely from the fact that most tumors have foci of hypoxic, yet presumably viable, cells, and it is commonly thought that it is the se foci that make it difficult to control a number of tumors adequately with conventional low-LET radiation therapy. Since the effects of high-LET radiation are much less dependent on oxygen tension than are those of conventional radiations, they may have the potential for more effective tumor control. However, one obviously needs penetration of many centimeters, obtainable only with heavy ions accelerated to energies of several hundred $\mathrm{MeV} / \mathrm{amu}$ or more.

Another important characteristic of heavy particle beams that makes them attractive for radiotherapy is the precision with which energy car be deposited in tissue. For a given dose to the tumor in depth, one must, with gamma or neutron radiation, accept a relatively high dose to intervening normal tissues and to skin. With protons, alpha particles, heavy ions and pions, however, the dose to normal tissues for the same dose to the tumor is appreciably smaller. In addition, with gamma rays or fast neutrons, it is necessary to accept a sizable dose to tissues distal to the tumor, whereas, with particle beams, a relatively well defined range exists which allows one to stop the beam short of vital normal structures. Also, with gamma rays or neutrons, because of unavoidable penumbra effects, normal tissues lateral to the path of the beam receive relatively large doses. With heavy particles, good lateral distributions are achievable because of the vary small angles of scattering of these particles. 
The degree to which heavy ions may prove to be useful in radiotherapy depends on factors as yet insufficiently evaluated, namely the relative and absolute degree to which improvement in depth dose patterns and OER* increase the ability to control tumors. Should improved depth dose alone be shown to be of overriding importance, there would be little interest in the use of heavy ions since depth dose patterns comparable or superior to those of heavy ions can be achieved with profions, alpha particles or pi minus mesons. Should improved OER alone turn out to be of major importance, then there should be great interest in heavy ions. It is probable (but not yet shown) that OER values obtainable with energetic heavy ion beams may be very much more favorable than those obtainable with fast neutrons, or in the "star" region of a pion beam. The relative importance of depth dose and OER can best be evaluated by separate therapeutic trials using protons (or alpha particles) and fast neutrons, respectively.

* There is some evidence that OER may not be the only or principal reason that high-LET radiations appear to be more effective than low-LET radiations for the control of tumors. This is in a sense academic, since fast neutrons seem to be superior, whatever the reasons may be. 


\section{EXISTING HEAVY ION FACILITIES}

Presently many operating nuclear physics accelerators provide heavy ions for investigations in a number of scientific disciplines; nuclear, solid state and atomic physics, material science and biomedicine. Their increasing usage for this purpose reflects high scientific interest in these research areas based upon the results already obtained and which convincingly attest to the richness of the phenomena that such research can uncover. This has led to an increasing realization among American scientists of the need for the construction of facilities which will exploit these possibilities. Extensive construction activities underway in England, France, West Germany, and the Soviet Union will provide a broad ranging capability in heavy-ion science. In this section present facilities both here and abroad will be briefly described.

\section{A. United States Facilities}

The accelerators which are being used for heavy ion research are of various types: electrostatic accelerators, cyclotrons, linear accelerators and synchrotrons. According to the U.S. Atomic Energy Commission Report of November 1972 entitled "Heavy Ion Science, Research, Facilities, and Outlook", in fiscal year 1972 the operating costs of AEC supported heavy ion research were about $\$ 13,226,200 ; 256$ scientific man-years were devoted to this effort: To those estimates one should add the modest support of heavy ion research by the National Science Foundation.

\section{Electrostatic Accelerators}

The tandem electrostatic accelerators comprise a large fraction of the broad base of accelerator facilities engaged in nuclear research in the United States. This type of accelerator has been commercially available for almost two decades. It has been, in various models and arrangements, an important facility for both university-based and national laboratory-based research programs. The tandem electrostatic accelerator is eminently suitable for precision nuclear research investigations. It delivers a high quality, well collimated beam which can be readily varied in energy. An energy resolution of one part in $10^{4}$ is achievable. The tandem electrostatic accelerator generally operates with the ion source at ground potential. In this arrangement, negative ions are injected, accelerated back to ground potential. In a threestage mode of operation the ion source is placed in the terminal of a second electrostatic accelerator operating at a negative potential. Such an arrangement provides beams of higher energy. These accelerators are readily adapted for accelerating heavy ions. It is no surprise, therefore, that with the high interest in heavy ion research almost all the tandem electrostatic accelerators in the United States devote a significant and rapidly growing fraction of beam 
time to heavy-ion studies.

The double Emperor tandem Van de Graaff system at the Brookhaven National Laboratory is the highest energy electrostatic accelerator system in operation in the United States, or for that matter in the world. Although this facility is almost exclusively used for heavy-ion research studies, it is restricted to investigations in the low mass part of the periodic table. Indeed, the energies attainable exceed the Coulomb barrier for like target and projectile only up to about mass 44 . It follows that other electrostatic accelerators, many of which are also highly committed to heavy ion research, are limited to even lighter parts of the periodic table. A list of major electrostatic accelerators involved in heavy ion research follows -- very roughly (the se numbers serve only as a guide since there are substantial individual variations) the terminal voltage of an MP tandem is $10 \mathrm{MV}$, of an FN $7.5 \mathrm{MV}$, and of an EN $6 \mathrm{MV}$ :

\begin{tabular}{|c|c|}
\hline Double MP Tandems: & Brookhaven National Lab. \\
\hline MP Tandems & $\begin{array}{l}\mathrm{U} \text {. of Minnesota, } \mathrm{U} \text {. of Rochester, } \\
\text { Yale U. }\end{array}$ \\
\hline Double FN Tandem : & U. of Washington \\
\hline $\begin{array}{c}\text { Negative Injector, } \\
\text { FN Tandem }\end{array}$ & Los Alamos Scientific Lab. \\
\hline Double EN Tandem: & $\mathrm{U}$. of Pittsburgh \\
\hline $\begin{array}{c}\text { Negative Injector, } \\
\text { EN Tandem }\end{array}$ & $\mathrm{U}$. of Texas \\
\hline FN Tandems & $\begin{array}{l}\text { Argonne National Lab., Florida } \\
\text { State U., U. of Notre Dame, } \\
\text { Rutgers, Stanford, State U. of } \\
\text { N.Y. at Stonybrook, Triangle U. } \\
\text { Nuclear Lab., Purdue, Edgewood } \\
\text { Arsenal }\end{array}$ \\
\hline EN Tandems & $\begin{array}{l}\text { Lawrence Livermore Lab., Oak } \\
\text { Ridge National Lab., Cal. Inst. } \\
\text { of Tech., Kansas State U., U. } \\
\text { of Penna., U. of Wisconsin, } \\
\text { Rice, Western Michigan U. }\end{array}$ \\
\hline
\end{tabular}




\section{Eyclotrons}

A number of variable-energy, multiparticle cyclotrons have been constructed in the United States. These accelerators have initially all engaged in light ion nuclear research. Several of them, however, were designed with the intent to move into heavy ion acceleration and have done so. The two major designs which are being used for heavy ions in the United States are the 88-inch cyclotron design of the Lawrence Berkeley Laboratory and the 76-inch cyclotron design of the Oak Ridge National Laboratory. The cyclotron facilities at both of these laboratories are now heavily committed to heavy ion research.

The heavy ion energy available from a sector focused cyclotron is given approximately by the expression $T=k Q^{2} / A$ where $T$ gives the particle energy, $Q$ is the ion charge state, $A$ is the mass number of the projectile and $k$ is a constant for the particular cyclotron. The constant $k$ has a value of about $140 \mathrm{MeV}$ for the 88-inch cyclotron at Berkeley and a value of $90 \mathrm{MeV}$ for the 76-inch cyclotron at Oak Ridge. Attainable heavy ion beam energies from these cyclotrons exceed those from the Brookhaven double

- Emperor system, but the extension of the range of the periodic table opened for nuclear investigations is slight. Cyclotrons have the capability of providing intense beams and are already fulfilling an important role for a large class of heavy ion research investigations. Table 1 provides a list of typical heavy ion beams presently available at the 88 -inch cyclotron. Note that the external currents produced decrease rapidly as the mass number increases.

Texas A \& M University has constructed an 88-inch cyclotron of the Berkeley design and this facility is now also heavily engaged in heavy ion research. A 76-inch cyclotron of the Oak Ridge design has been constructed at the University of California at Davis. It currently accelerates some lightheavy ions but will have to undergo an improvement program to achieve heavyion beams comparable to those available from the Oak Ridge cyclotron. The 105-inch cyclotron at the $U$. of Maryland is another facility which possible. could be converted to heavy ion research. Other cyclotrons which may be utilized for heavy ion research are at Michigan State University (69"), Princeton University (69"), the University of Michigan (83") and Washington University (54").

\section{3. $\mathrm{RF}$ Linear Accelerator}

It is amusing to realize that the first rf particle accelerator was a linear accelerator and the first particles accelerated by it were heavy ions. Over 20 years later, in 1954, groups at Yale and the Lawrence Radiation 
TABLE I

Typical 88-inch Cyclotron Heavy Ion Beams (June 1973)

(This table furnished by Bernard G. Harvey)

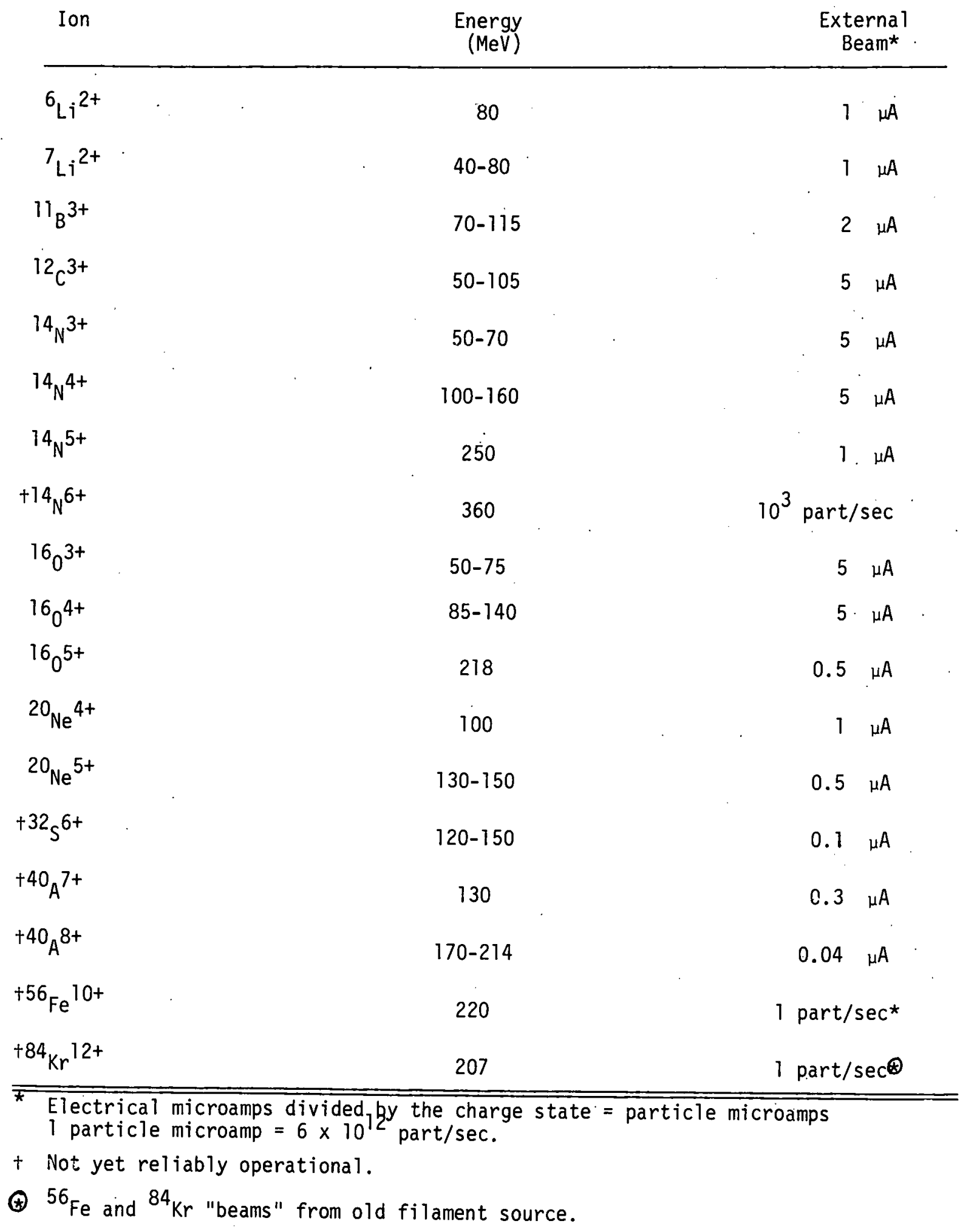


Laboratory completed a joint design of a heavy ion linear accelerator (HILAC) and finished the construction of essentially identical machines by 1957 . In this design a heavy ion beam is pre-accelerated in a Cockcroft-Walton and then injected into the pre-stripper linac. Upon emerging from this linac the beam is passed through a stripper, where the ions are further ionized. They then enter the post-stripper linac emerging with an energy of $10 \mathrm{MeV} / \mathrm{nucleon}$. The HILAC's produce useful beams of heavy ions with atomic masses up to $40{ }^{4}$.

A recent effort has been mounted to improve the Berkeley HILAC so as to provide beams of heavy ions of all species with a maximum energy of $8.5 \mathrm{MeV} /$ nucleon. This accelerator called the SuperHIIAC is expected to provide beams with currents varying from $10^{11}$ to $10^{15}$ particles/sec according to the nucleus being accelerated (see Fig. 2 and Table 2). Toward this end the HILAC has been rebuilt almost in its entirety. The same scheme of two linear accelerators separated by a stripper was retained but their design was based on a much smaller charge to mass ratio appropriate for the heavier ions. Two injectors are used. The first is a modified version of the original HILAC injector system having a terminal voltage of $800 \mathrm{KV}$ and is being employed to accelerate heavy ions up to ${ }^{40} \mathrm{~A}$. This component of the SuperHILAC is completed and has achieved reliable performance. For heavier nuclei, 2.5 MV dynamitron is used as an injector. The dynamitron has the troublesome feature that the heavy ion source is placed in the high voltage terminal. As of the present time, this high energy injector system is not operating properly although in May 1973, $\mathrm{Kr}$ beams were successfully accelerated several times for periods of 48 hours. Currents obtained at that time, together with the eventual expected current, are shown in Table 2 .

\section{Synchrotron}

Two synchrotrons, the Berkeley Bevatron and the PrincetonPennsylvania Accelerator, originally built to provide high energy beams of protrons of $6.2 \mathrm{GeV}$ and $3 \mathrm{GeV}$ respectively, have been modified to allow acceleration of heavy ions. The PPA successfully accelerated nitrogen ions before it was shut down at the end of FY 71. The Bevatron has circulated beams of ${ }^{12} \mathrm{C},{ }^{14} \mathrm{~N},{ }^{16} \mathrm{O}$ and ${ }^{20} \mathrm{Ne}$ to energies of $2.5 \mathrm{GeV} /$ nucleon. These were extracted and delivered to targets set up in the external beam lines. Typical beam current (particles/pulse) in use at the Bevatron as of June 1973 are given in Table 3. (Approximately 25\% of the Bevatron operation time is now being used for heavy ion research and development. Experiments have been made with relativistic ions in the fields of high energy physics, nuclear structure, cosmic rays and biomedical research.) 
TABLE 2

SuperHILAC Beams

(Integrated particle currents)

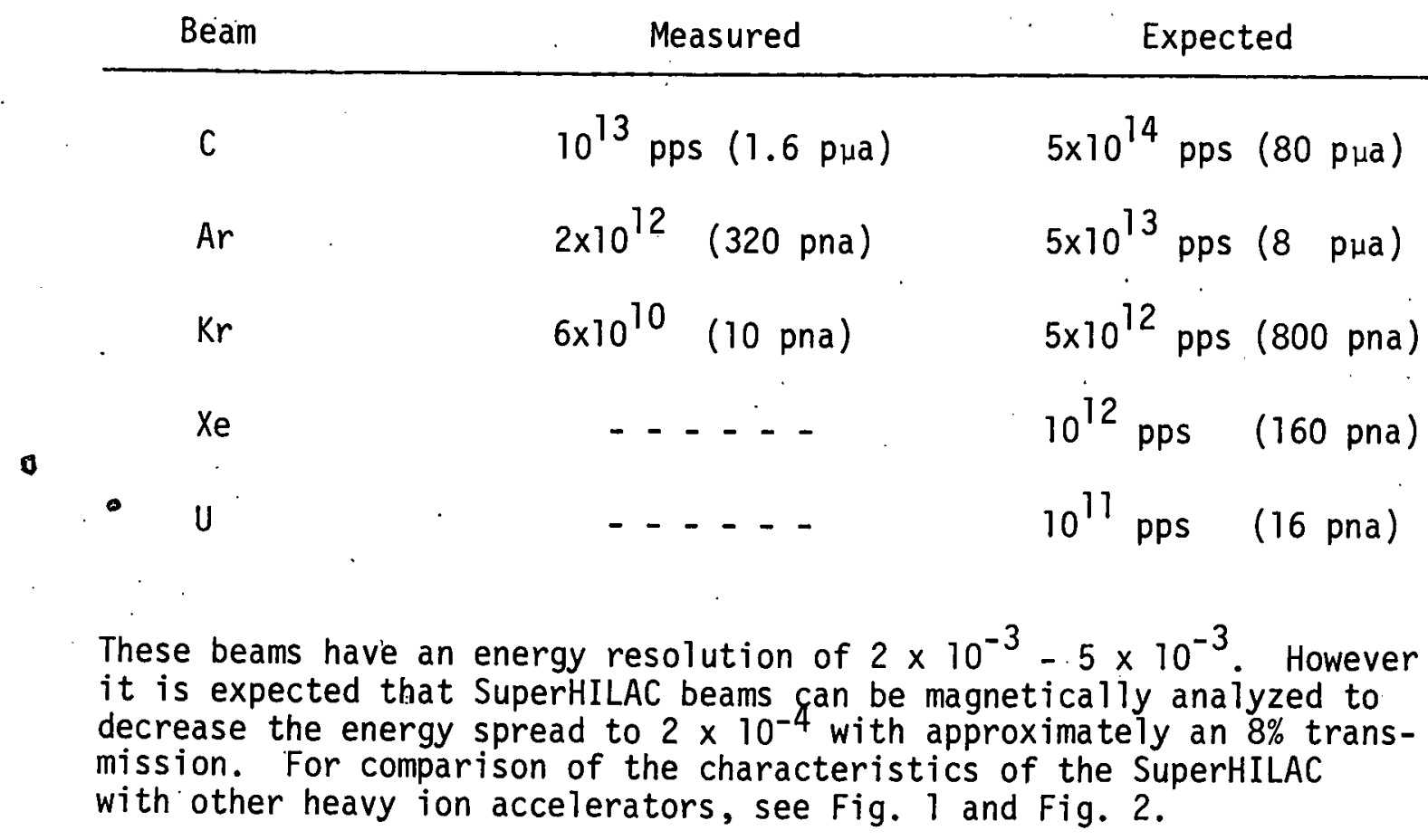

TABLE 3

Intensity of Bevatron Heavy Ion Beams (June 1973)

Ion

(Particles/pulse) on Target*

${ }^{12} \mathrm{C}$

$1 \times 10^{8}$

${ }^{14} \mathrm{~N}$

$1 \times 10^{7}$

$16_{0}$

$1.5 \times 10^{7}$

$20 \mathrm{Ne}$

$10^{5}$

* There are 10-17 pulses per minute. 


\section{B. Heavy Ion Accelerators Outside the United States}

The opportunities provided by heavy ion physics have long been recognized by European physicists and their supporting agencies leading tó today's intense activity, both in research and in the construction of new facilities. New facilities are being built in West Germany (UNILAC) and Great Britain (DARESBURY). France, which already has an excellent facility in ALICE, is planning to build one of greater capability, GANIL. The Chalk River Laboratory of the Atomic Energy of Canada Ltd . is about to propose an Option I facility. The Soviet Union has a facility at Dubna, which has for some time had the heaviest ion beam in the world, which can penetrate the Coulomb barrier of uranium. To these facilities one should add the European tandem accelerators and cyclotrons which,like their United States counterparts, spend much of their operational time on heavy ion physics. In addition, advanced tandems which are to be used for heavy ion research are being installed in Australia and Israel and are being actively considered in Japan.

In Fig. 1 and Fig. 2 , the characteristics of the se heavy ion facilities are compared with each other and with the SuperHILAC and the Oak Ridge proposal for a National Heavy Ion Laboratory. In what follows, the se European proposals will be described to the extent that our incomplete information permits. It is, however, abundantly clear that European physicists have decided to put a massive effort into heavy ion physics.

\section{UNILAC (West Germany)}

This accelerator is under construction. It is expected to begin operation early in 1975, providing beams with full energy by the end of 1975 . The accelerator itself cost $47 \times 10^{6} \mathrm{dM}$, the building housing the accelerator and experimental areas etc., $66 \times 10^{6} \mathrm{dM}$, the ancillary equipment and computer $29 \times 10^{6} \mathrm{dM}$. These sums total to $142 \times 10^{6} \mathrm{dM}$.

The accelerator is a linac. The ion source is at $320 \mathrm{KV}$, the first linac section (Wideroe) delivers ions of $1.4 \mathrm{MeV} /$ nucleon which strike a stripper and then enter the second section consisting of two Alvarez type linacs, leaving that section with $5.9 \mathrm{MeV} /$ nucleon. The third section is made up of 20 single resonators which can be separately tuned. Depending upon the type of stripper (gas or solid) the final ion beam energy is $8.5 \mathrm{MeV} / \mathrm{nucleon}$ or 10.2 MeV/nucleon respectively. The solid stripper curve is shown in Fig. 1.

The projected currents are given in Fig. 2. For the lower masses, the duty cycle is $100 \%$, while for the heaviest ions it is about $25 \%$. If ions with several charge states are accelerated, the energy resolution of the ion beam is $10^{-2}$; if ions with one particular charge state are selected, it is $10^{-3}$. The energy resolution can be improved to $2 \times 10^{-4}$, by analys is after the beam leaves the linac. A full complement of ancillary apparatus will be available 


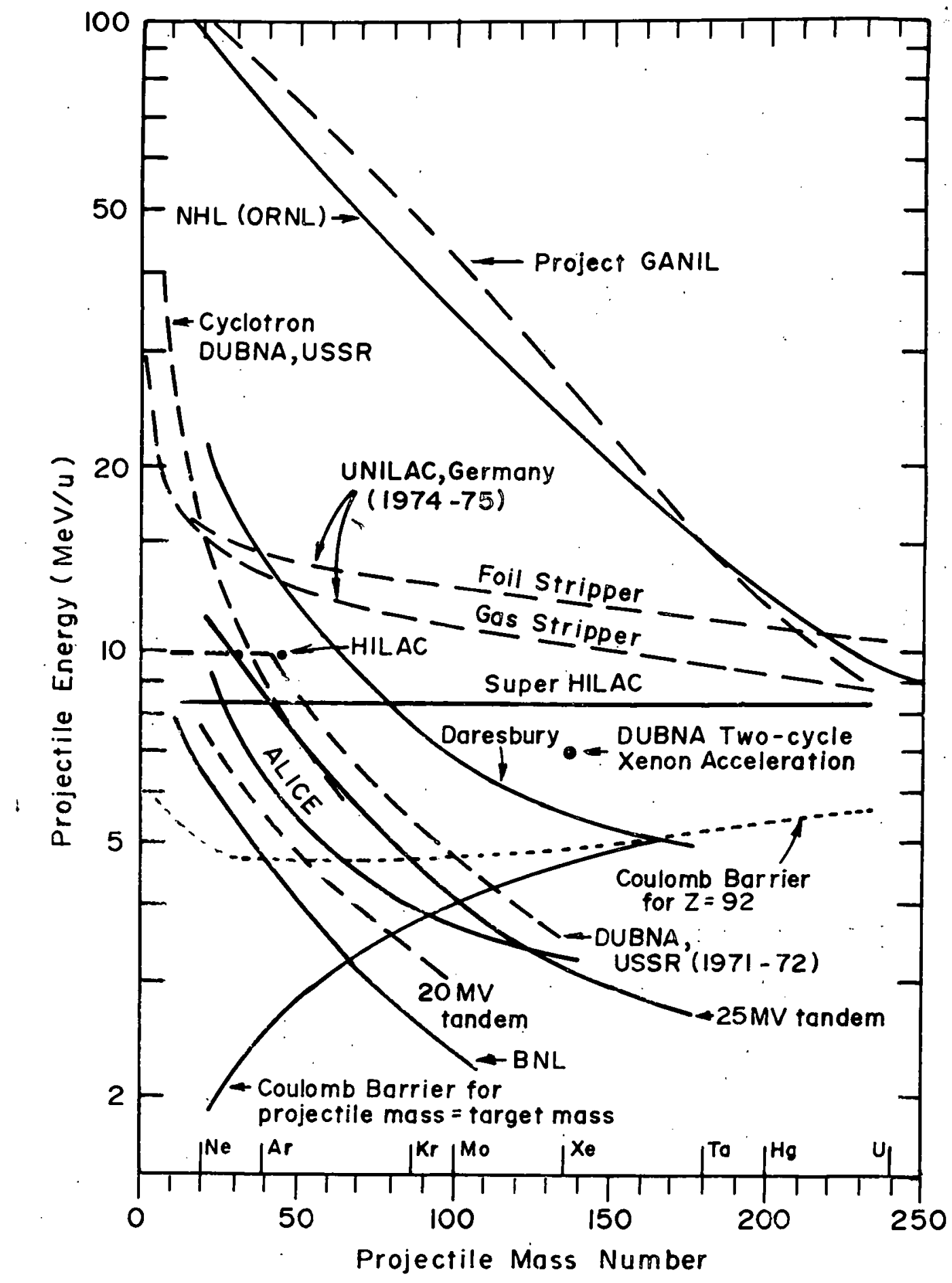

Figure 1 


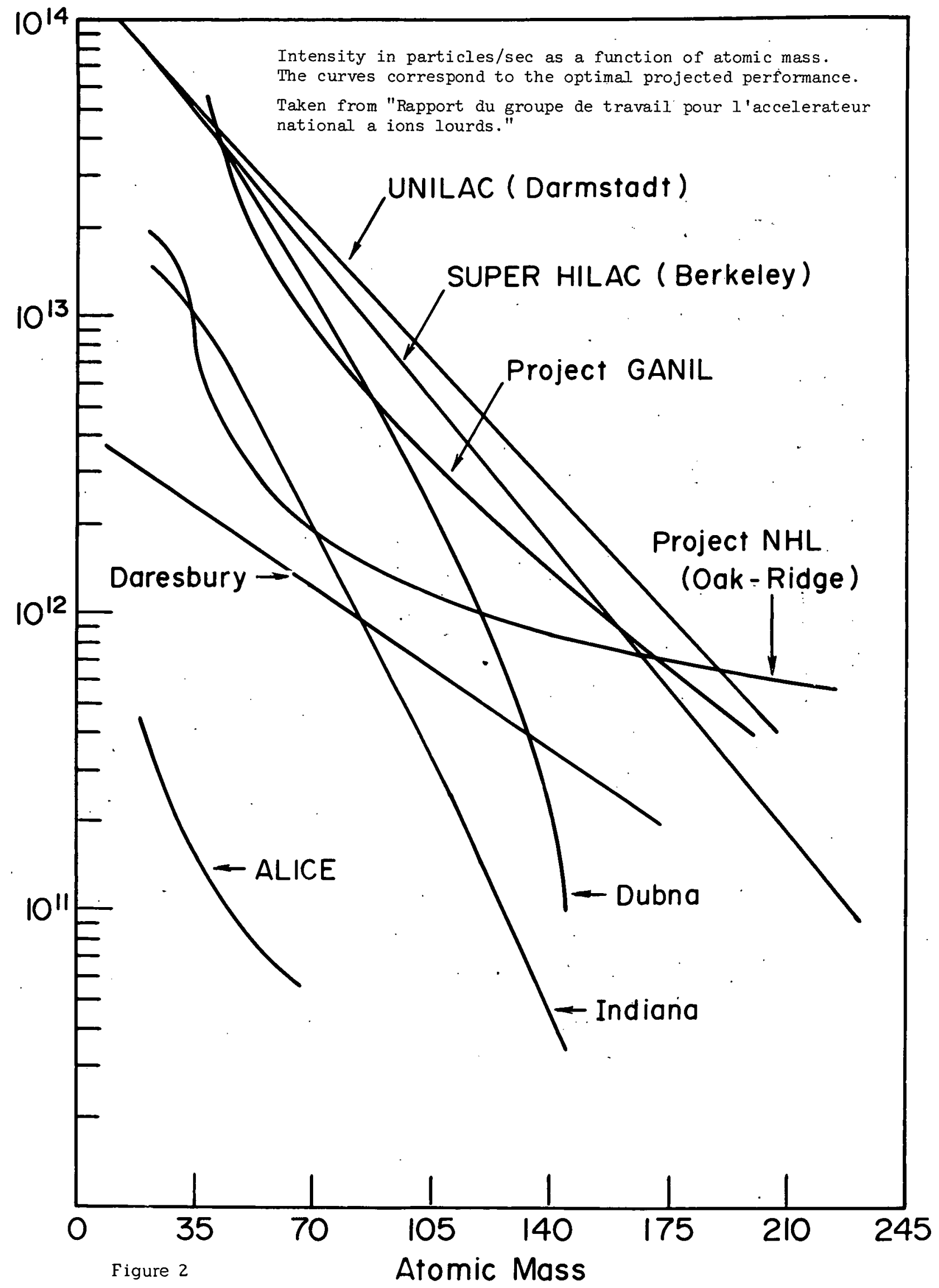


including a velocity selector to separate reaction products from the incident beam and an on-line isotope separator. Provision is made for fast chemistry . In addition to scattering chambers, provision has been made for $x$-ray and $\gamma$-ray spectroscopy. A time of flight telescope, a magnetic spectrometer, a wide angle detector and a position sensitive detector are planned. Planning is underway for probable upgrading through provision of a high energy tandem electrostatic accelerator as an alternate injector.

\section{ALICE and GANIL (France)}

The heavy ion accelerator, ALICE, at Orsay is operational. The beam from the injector enters a linac section leaving with an energy of $1 \mathrm{MeV} /$ nucleon. This beam is transported from the linac and injected into a isochronous cyclotron. Carbon foil strippers are placed within the cyclotron. The heaviest ion for which this accelerator is planned, $\mathrm{Kr}$, acquires an energy of $440 \mathrm{MeV}$ close to the Coulomb barrier for the $\mathrm{Kr}+\mathrm{Kr}$ reaction. The energy and current provided by ALICE are shown in Fig. l and Fig. 2. The macroscopic duty cycle is $25 \%$.

The French have recently completed an indepth conceptual study (lasting about 6 months) of their next heavy ion accelerator. The actual design phase of the study, which has already begun, is expected to be completed in 1974 . They hope to finish the accelerator by 1980. They estimate their cost to be $150 \times 10^{6} \mathrm{fr}$. , of which $70 \times 10^{6}$ will be for the accelerator complex, the remainder for the building and equipment. As can be seen from Fig. 1 and Fig. 2, the planned energy/nucleon will for all ions exceed $10 \mathrm{MeV} /$ nucleon. In light ions, the energy will be approximately $100 \mathrm{MeV} /$ nucleon, decreasing with increasing mass. They plan larger currents for the light ions, about $10^{12}$ part/sec, and smaller currents for the heavier ions, about $10^{11} \mathrm{part} / \mathrm{sec}$. The energy resolution will be $2 \times 10^{-4}$ for the lighter ions (A 40) and $10^{-3}$ for the heavier ones. Duty cycle will be close to $100 \%$ for the light ions and between 25 and $50 \%$ for the heavier ions.

The system will consist of two separated sector cyclotrons, $(k=380)$ with a solid stripper between. They feel it is very important to obtain very energetic light ions and, for that reason, did not use the linear accelerator. Provision is also made for the installation of a tandem which could inject into one of the cyclotrons, but this system does not have the first priority. One of their objectives is to have an accelerator whose capability will differ from that of the UNILAC.

Among the ancillary equipment envisioned, is a high resolution magnetic spectrograph, a spectrograph for the very heavy-ions, an on-line spectrograph for short-lived isotopes, and a number of scattering chambers. The cost of this equipment is estimated to be $15 \times 10^{6} \mathrm{fr}$. 


\section{Dubna (Soviet Union)}

The heavy ion facility at Dubna consists of two cyclotrons, one of $310 \mathrm{~cm}$ radius which is used to inject into the second $200 \mathrm{~cm}$ radius cyclotron. This system has been used to accelerate Xe beams. At the end of the first stage $\mathrm{Xe}^{8+}$ has been accelerated to an energy of $0.92 \mathrm{MeV} /$ nucleon. This beam is then stripped by a carbon foil. The $\mathrm{Xe}^{28+}$ ion is selected and accelerated in the second cyclotron. The final beam current is $2 \times 10^{10}$ particles/sec of $\mathrm{Xe}$ ions having an energy of $7 \mathrm{MeV} /$ nucleon. For characteristics of the Dubna accelerator see Fig. 1 and Fig. 2 .

As was mentioned earlier this $\mathrm{Xe}$ beam is the heaviest ion beam available today which can penetrate the Coulomb barrier of uranium. The Dubna group are in fact in the process of studying the interaction between these ions and uranium, as well as tellurium.

There is no question that heavy ion physics and in particular the search for new elements is a most strongly supported segment of the Soviet program in nuclear physics.

\section{The DARESBURY Accelerator (Great Britain)}

The construction of this accelerator has been approved by the Science Research Council. It is a tandem electrostatic accelerator with $20 \mathrm{MV}$ terminal and voltage with the possibility of upgrading to $30 \mathrm{MV}$. This will not be a commercial machine. Several new elements in the accelerator design will require development; such a program is now in progress. A number of ion sources are planned, the desired ion being selected by an analyzing magnet. A similar magnet is used to deflect the emerging beam into one of a number of beam lines. There is provision for three strippers, two of which are foil strippers. The third, located in the center terminal, may be either gaseous or foil as required. The cost of the accelerator, including ion source and its injection, beam handling and beam lines, some initial experimental equipment (some to be taken from other laboratories) including a computer, and a building including three experimental areas, is approximately $£ 4.7 \times 10^{6}$. The proposal recognizes that the ancillary equipment provided in this plan is inadequate and plans later additions. Space has been reserved for the possible addition of an isochronous cyclotron or linac into which the tandem beam could be injected. Operating çosts are uncertain but as a minimum they are expected to be $£ 540 \times 10^{3}$. The construction time is estimated to be 4.5 years. The characteristics of the DARESBURY machine are shown in Fig. 1 and Fig. 2. Beams of masses up to about $A=140$ can overcome their own Coulomb barrier while masses up to about $A=120$ can penetrate the Coulomb barrier of uranium. 


\section{Chalk River (Canada)}

The Chalk River Laboratory of the Atomic Energy of Canada, Ltd. is presenting a proposal calling for a superconducting cyclotron injected by their existing $13 \mathrm{MV} \mathrm{Mp}$ tandem electrostatic accelerator. The cyclotron would have a $k$ of $507\left(E=k Q^{2} / A\right)$ and a radius of $65 \mathrm{~cm}$. It is designed to produce uranium ions with $10.35 \mathrm{MeV} /$ nucleon with currents of greater than $10^{12}$ particles/sec. The expected energy resolution is $10^{-4}$. The cost of the cyclotron, refrigeration and controls is estimated to be $\$ 2.7 \times 10^{6}$. The cost of the entire installation is not known to the Panel.

\section{Israel}

The Weizman Institute group in Israel has purchased a $14 \mathrm{MV}$ tandem accelerator with extensive-additional components designed to optimize heavy ion performance. The group is engaged in a joint study with one at Stanford University directed toward provision of a superconducting linear accelerator booster to be injected by the tandem. Building plans for the two accelerator complex are complete and the project appears to have strong governmental support. The total cost is not known to the Panel. 


\section{ACCELERATOR AND ANCILLARY EQUIPMENT CHARACTERISTICS}

The scientific and technological goals outlined in Section III imply certain requirements for the accelerator complex and the associated ancillary equipment. In the sections which follow the required facilities will be described for each field of interest.

\section{A. Characteristics Required for Physics and Chemistry Research}

The experimental programs in these fields generally require sufficiently intense beams of a broad variety of ions from the light ions and extending. up through the periodic table to uranium. Ion energies should extend considerably above the Coulomb barrier. In this way, collisions between a variety of types of nuclei can be studied and kinematic conditions varied so that "windows" covering a wide range of energies and angular momenta can be explored. It is thought that the chances for the formation of superheavy nuclei would be enhanced if the mass of the projectile differs substantially from that of the heavy target nucleus. These opportunities can only be realized if the energy of the heavy ion can be continuously varied and if the beam projectile can be rapidly changed. In the non-relativistic domain, a nominal energy of $10 \mathrm{MeV} /$ nucleon has been used to indicate the order of magnitude of a reasonable energy. Uranium ions with this energy will be able to penetrate the Coulomb barrier of a uranium nucleus, which is about $6 \mathrm{MeV} /$ nucleon. It thus follows that Coulomb barriers will be exceeded for collision between any pair of stable nuclei. The optimal energy for relativistic heavy ions is not known; heavy ion beams of the one operating accelerator, the Bevatron, have an energy of $2.1 \mathrm{GeV} /$ nucleon.

The detailed criteria for energy and angular resolution and beam current differ according to whether the investigations are focused upon the macroscopic properties of nuclei and nuclear interactions or upon their quantal or microscopic properties. The former, the macroscopic, will generally require less resolution but more intense currents. For example, for isotope production the highest possible beam currents are desirable. These currents are limited essentially by target properties. A reasonable estimate is $10^{13}$ particles/ $\mathrm{sec}$ for the lighter ions and $10^{12} \mathrm{particle} / \mathrm{sec}$ for the heaviest ions. Energy resolution need only be about $10^{-3}$ for most cases although occasionally resolutions of up to a factor of five times better may be needed.

The investigation of the quantal properties puts considerably more stringent conditions on the heavy ion beams. 


\section{Resolution}

Clearly, if heavy ion beams are to be used to probe nuclear phenomena one of the requisites in large classes of experiments is that individual quantum states in residual nuclear systems be resolvable. While this requirement poses relatively few problems in interactions involving relatively light projectiles and targets (characteristic of much of the heavy ion reaction studies thus far published) because of the relatively large ( $\mathrm{l} \mathrm{MeV}$ ) level spacings involved, it becomes a much more severe experimental limitation as the atomic number of either the projectile or target is increased. In heavy nuclei, level spacings, even at low excitations, are typically $\sim 100 \mathrm{KeV}$ or less. Thus large classes of experiments, probably adequate to delineate broad ranges of phenomena, would be accessible in selected nuclear systems in experiments characterized by energy resolutions of $\sim 100 \mathrm{KeV}$.

Much has been written about the difficulties anticipated in attaining such resolutions in heavy ion reactions particularly in connection with the heavier ions where target problems become significant; problems such as energy straggling and multiple scattering in the target as well as beam heating of the target. These difficulties are real and limit the energy resolution that can be readily obtained for the heavier ions to approximately $10^{-3}$. However, if there are sufficiently important experiments that need to be done and which require better resolution there are techniques available which circumvent these target difficulties.

Major progress has been made in recent years in evolving mechanisms for the production of ultra thin solid targets. Areal densities in the range of $10 \mathrm{micrograms} / \mathrm{cm}^{2}$ have been attained for self-supporting solid targets of several elements throughout most of the periodic table. Production of very thin films of gas, cryogenically cooled just to the condensation point and released from appropriate nozzles above the projectile beam so that a continuously renewed target film falls across this beam (later trapped by freezing out on cryogenic surfaces below the beam), have already been demonstrated as feasible for the production of ultra thin targets--a few micrograms $/ \mathrm{cm}^{2}$-for all species available initially in gaseous form. In addition, it should be emphasized that a flexible heavy ion accelerator, wherein beam species can be selected widely, conveniently, and quickly can frequently avoid difficulties by ready interchange of target and projectile species to yield a more amenable target fabrication situation. It is not unreasonable to conclude then that with a few exceptions, targets with areal densities of the order of 10 micrograms per $\mathrm{cm}^{2}$ are conceivable. The fact that the yield from such targets, particularly in heavy ion reactions having a great many competing open reaction exit channels, can be expected to be very low can be partially compensated by arranging for mechanical rotation or oscillation of the target film during 
bombardment so that correspondingly higher beam intensities can be tolerated without target destruction.

It may be worth including a few illustrative state-of-the-art examples appropriate to either existing or projected studies with projectiles of low, intermediate and relatively heavy mass on a variety of targets.

\section{a. Light heavy ion projectiles}

i) Using ordinary surface barrier semiconductor detectors in the study of inelastic scattering of ${ }^{12} \mathrm{C}$ ions on ${ }^{152} \mathrm{Sm}$ at incident ${ }^{12} \mathrm{C}$ energies of $40 \mathrm{MeV}$, energy resolutions (FWHM) of $60 \mathrm{KeV}$ have been achieved in many laboratories.

ii) Using magnetic spectrographs and a $5 \mu \mathrm{g} / \mathrm{cm}^{2}{ }^{12} \mathrm{C}$ target, energy resolutions in the range $25-30 \mathrm{KeV}$ have been achieved in many laboratories in ${ }^{16} \mathrm{O}+{ }^{12} \mathrm{C}$ inelastic scattering studies at incident energies above, approximately, $35 \mathrm{MeV}$.

iii) Typically, without any special effort, energy resolutions less than $250 \mathrm{keV}$ are achieved routinely for projectiles lighter than neon.

\section{b. Medium mass heavy ion projectiles}

At low projectile energies target thickness imposes resolution limitations but at higher energies intrinsic beam energy resolution governs. The following table for ${ }^{32} \mathrm{~S}$ is illustrative and includes ${ }^{16} \mathrm{O}$ for comparison; nickel and gold targets are used for illustration (see Table 4). Since these are not limiting target thicknesses, it is clear that an intrinsic beam energy resolution of $10^{-4}$ will be essential to large classes of experiments .

\section{c. Higher mass heavy ion projectiles}

Two examples from transuranic work will suffice. At 12 $\mathrm{MeV} /$ nucleon $\left(672 \mathrm{MeV}\right.$ ), target thickness effects for the ${ }^{238} \mathrm{U}+{ }^{56} \mathrm{Fe}$ reaction are not significantly different from those noted above for the $16 \mathrm{O}+{ }^{12} \mathrm{C}$ studies. At this energy the iron beam loses only $9.5 \mathrm{keV}$ of energy per microgram of $\mathrm{UF}_{6}$ target thickness. 
TABLE 4

\begin{tabular}{|c|c|c|c|c|c|c|}
\hline $\begin{array}{c}\text { ION } \\
\text { SPECIES }\end{array}$ & $\begin{array}{l}\text { ION } \\
\text { ENERGY }\end{array}$ & $\begin{array}{l}\text { ENERGY } \\
\text { PER } \\
\text { NUCLEON }\end{array}$ & $\begin{array}{l}\text { ENERGY LOSS } \\
\text { IN } 10 \mu \mathrm{g} / \mathrm{cm}^{2} \\
\text { Ni TARGET }\end{array}$ & $\begin{array}{l}\text { BEAM RESOLUTION } \\
\text { FOR COMPARABLE } \\
\Delta E\end{array}$ & $\begin{array}{l}\text { ENERGY LOSS } \\
\text { IN } 50 \mu \mathrm{g} / \mathrm{cm}^{2} \\
\text { AU TARGET }\end{array}$ & $\begin{array}{l}\text { BEAM RESOLUTION } \\
\text { FOR COMPARABLE } \\
\qquad E\end{array}$ \\
\hline \multirow[t]{2}{*}{${ }^{32} \mathrm{~s}$} & $80 \mathrm{MeV}$ & $2.5 \mathrm{MeV}$ & $\sim 100 \mathrm{keV}$ & $1.25 \times 10^{-3}$ & $\sim 300 \mathrm{keV}$ & $\sim 4 \times 10^{-3}$ \\
\hline & $320 \mathrm{MeV}$ & $10 \mathrm{MeV}$ & $\sim 60 \mathrm{keV}$ & $2 \times 10^{-4}$ & $\sim 185 \mathrm{keV}$ & $6 \times 10^{-4}$ \\
\hline \multirow[t]{2}{*}{${ }^{16} \mathrm{O}$} & $48 \mathrm{MeV}$ & $3 \mathrm{Mev}$ & $\sim 38 \mathrm{keV}$ & $8 \times 10^{-4}$ & $\sim 105 \mathrm{keV}$ & $2 \times 10^{-3}$ \\
\hline & $160 \mathrm{MeV}$ & $10 \mathrm{MeV}$ & $\sim 18 \mathrm{keV}$ & $1 \times 10^{-4}$ & $\sim 55 \mathrm{keV}$ & $4 \times 10^{-4}$ \\
\hline
\end{tabular}


A favorite reaction now under study as a potential route to supertransuranic species is

$$
{ }^{76} \mathrm{Ge}+{ }^{232} \mathrm{Th}+{ }^{308} \mathrm{X}_{122}+{ }^{298} \mathrm{Y}_{114}+?
$$

At $12 \mathrm{MeV}$ per nucleon a ${ }^{76} \mathrm{Ge}$ beam loses $9.4 \mathrm{keV}$ per microgram of target material.

In both cases a beam resolution of $10^{-4}$. could be exploited.

\section{Variability}

During the past year rather surprising results in several laboratories have shown that relatively sharp states ( $\Gamma^{\sim}$ few hundred keV) exist at excitations of $\sim 40 \mathrm{MeV}$ in medium mass nuclei where they appear as resonances in appropriately selective heavy ion reactions. The se are among the most interesting new phenomena in heavy ion science inasmuch as they provide access to a whole new class of nuclear structures and, on the basis of preliminary evidence, whole new parentage families in adjacent nuclei at these unexpectedly high excitations .

Such phenomena would be totally inaccessible without heavy ion beams having simultaneously high intrinsic energy resolution $\left(210^{4}\right)$ and convenient and rapid energy variability so that excitation functions (variations of reaction cross sections with energy) may be traced in detail and without requiring prohibitively large allocation of accelerator time. As yet this is an almost completely new and unexplored field; what has been found, with each new generation of accelerator capable of providing yet higher energy heavy ion beams with adequate energy resolution, is that totally unexpected sharp structure has been found up to the available energy limit in the measured excitation functions. There is little reason to anticipate that this will not continue to be the case with the next generation of higher energy facilities.

In the case of supertransuranics, it may well be that the current structure calculations give an optimistic estimate of the half lives against decay. (These range from a few months to $10^{5}$ years for $\mathrm{Z}=114$, for example, depending upon the details of the potential and model used to extrapolate from known level characteristics of the lead region into the supertransuranic region.) It may well be that the supertransuranic species will only be observable as compound system resonances in suitably chosen heavy ion reaction systems. In order to hope to observe such resonances, the combination of high energy resolution and easy variability is mandatory.

Nuclear physics studies involving light projectiles at energies available to precision studies, have been characterized by an early focus on angular distributions -- the variation of reaction cross sections with angle of observation. This occurred naturally as a consequence of the fact that these 
reactions involved a very limited number of partial waves, in general, and, in particular, because these angular distributions typically showed strong sensitivity to, and discriminated among, different values of angular momentum transferred in the reactions.

However, it was one of the significant discoveries of the last decade that very important phenomena could be uncovered if the energy dependence of the reaction cross sections--excitation functions--were studied. These experiments required high resolution and energy variability as the structures in energy being sought for often have narrow widths and need to be seen against a background of real, but less interesting, events. It should be emphasized that the discovery of these structures is a most important goal of nuclear physics. They are a clear manifestation of the characteristic modes of motion of nuclei. The strong interest in heavy ion nuclear physics originates in great part from the expectation that in the collision of heavy ions with nuclei, such fundamental nuclear properties will be most prolifically revealed.

Attention is thus increasingly turning toward exploitation of excitation functions as the most physically significant measurements. And just as in the case of the angular distributions, where nuclear physicists eventually learned that attempts to understand isolated measurements at only a few energies were essentially meaningless, so also in the case of excitation functions has it been found essential to measure these at many angles of observation.

All this puts a high premium not only on having adequate beam energy resolution to permit delineation of fine structure in the excitation functions (e.g., resonances and potential scattering structure), but also in being able to change the output energy of the accelerator and the beam transmission systems in periods to be measured in terms of a few minutes at most, if such measurements are to be accomplished in reasonable time span and overall accelerator and facility operational costs. In a study such as that in ${ }^{16} \mathrm{O}+{ }^{16} \mathrm{O}$, for example, experimental points have typically been measured at $100 \mathrm{keV}$ intervals in the range from 6 to $70 \mathrm{MeV}$ and more recently $\sim 100 \mathrm{MeV}$. With 1000 points these are already lengthy measurements. With semiconductor detectors many angles are studied simultaneously but where magnetic spectrographs or spectrometers are required, unless a large multigap facility is available, the required time goes up directly with the number of angles involved. Beam energy change time of $\sim 0.5$ to 1 hour would be totally unacceptable in such studies.

It must be emphasized that any accelerator can be made to produce an output beam of any desired energy resolution provided that arbitrary fractions of the total beam can be thrown away in its definition (with consequent dwind- 
ling useful intensity) and provided that arbitrary amounts of time are available for the fine tuning of the beam analysis and transport systems which may be involved. However, such strategems are of importance only if they can be achieved and are essentially meaningless if the objective cannot be attained with an intensity and in an adequately short time interval to make it useful in a realistic experimental contex.

It is in just this context that the high degree of complementarity between facilities such as SuperHILAC and a very large electrostatic accelerator becomes most evident. Whereas the former characteristically provides higher energies and intensities, the latter provides higher resolutions and ease of energy variability.

\section{Angular Resolution}

The recent discovery of diffraction oscillations in particle transfer and inelastic excitation as well as the more familiar example of elastic scattering underline the need for good angular resolution. These diffraction patterns are sensitive not only to the size of the colliding nuclei but also their surface properties, the absorption part of the interaction between them and in the case of inelastic scattering, to the quadrupole moment of the excited state. The angular resolution which is needed for the determination of the se phenomena must improve as the energy or mass of the interacting nuclei increases. Angular resolutions of the required order of magnitude are readily obtainable for heavy ion projectiles with atomic number, $Z$, less than fifty. For more massive projectiles, target problems of the type discussed above in the section on energy resolution become serious. As in that situation, angular resolutions which will permit the detection of diffraction structure can be obtained if needed but only when special measures are taken. These will generally be undertaken only if especially important scientific issues are involved.

\section{B. Characteristics Reguired for Biomedical Research}

For use in radiotherapy research, the basic requirements are for fully stripped ions of $Z$ up to 18 accelerated to energies in the range of approximately $1 \mathrm{GeV} / \mathrm{amu}$ or greater. The intensity should be such that dose rates can be achieved of the order of 40 to 100 rads per minute, over a tumor

treatment volume of approximately $1,000 \mathrm{~cm}^{3}$. These characteristics would allow one to obtain the depth of penetration necessary to irradiate deep-seated tumors of sizes frequently encountered. They would also allow one to obtain the high values of LET that may result in an oxygen enhancement ratio (OER) sufficiently low to aid materially in overcoming the problem of hypoxic cells in tumors. 
The necessary radiobiological work to determine how attractive these ion beams may be for radiotherapy has not been completed, and it is quite important that these studies be carried out. Included are investigations at the cellular level of dose-effect relationships, cellular repair capability, the RBE, and the OER. Also required are studies at the tissue level, to determine the degree to which tissues can recover from the effects of high LET radiation, and to determine if unanticipated early or late effects may be associated with the use of these radiations. More studies are required to determine the degree to which heavy ions induce cancer.

It is also necessary to do detailed measurements to determine the dose distribution both in depth and laterally. Such measurements are necessary to determine the precision with which the energy can be deposited in the treatment volume, and to determine the ratio of dose to the tumor relative to that received by normal tissues.

It appears reasonable that the above indicated studies can be done with a single facility having the capabilities outlined above. If, as a result of the evaluative studies, it is determined that the beam is indeed attractive for radiotherapy, then consideration must be given to the construction of additional "dedicated" facilities to allow adequate clinical evaluations of the efficacy of this treatment modality, and to take full advantage of its potential in radiotherapy.

With respect to evaluating the possible hazard to astronauts from high energy stripped nuclei in space, it is also necessary to have available stripped particles up to $Z$ of at least 18 and preferably 26 , accelerated to energies high enough to insure penetration of several centimeters in tissue. It would be very difficult if not impossible, to do the proper evaluative studies with beams of stripped heavy nuclei of energies less than several hundred $\mathrm{MeV}$ per nucleon.

Some, but not all of the dosimetric and radiobiological studies required to evaluate the efficacy of heavy particle beams in radiotherapy could be done with beams of particles in the $10^{\prime} \mathrm{s}$ to perhaps $100 \mathrm{MeV} / \mathrm{amu}$ range. However, since all of these studies can be done more easily and efficiently with the very high energy particles, there appears to be no compelling requirement for particles of lesser energy for this purpose.

Basic radiochemical and radiobiology studies can be done even with beams with energies as low as $10 \mathrm{MeV} / \mathrm{amu}$. However, investigative work becomes increasingly more difficult as the energy is reduced. In the energy range of $10 \mathrm{MeV} / \mathrm{amu}$ one is limited to living systems that can be mounted in a monolayer or in a thin wafer of the order of 100 microns thick. This severely 
restricts the types of biological materials that can be used. Also, the beam must be external, since it is only under rare circumstances that one can work with biological specimens in a vacuum. Particles in the energy range of $100 \mathrm{MeV} /$ nucleon would be of considerably more interest for radiobiological studies, since one can attain penetration in tissue of the order of millimeters to centimeters, depending on the $\mathrm{Z}$ of the particle used.

There is little question that important radiobiological and radiochemical work could be done if additional facilities became available that were capable of accelerating heavy ions to energies in the range of $10^{\prime} \mathrm{s}$ to $100 \mathrm{MeV} / \mathrm{amu}$. However, it does not seem reasonable at this time to consider this potential use as a substantial part of the overall justification for the construction of such a facility.

\section{Characteristics Required for Atomic Physics}

The widest possible range of ion species is needed for atomic physics. To investigate highly stripped atomic systems the highest energy possible is desirable. At $10 \mathrm{MeV} /$ nucleon it will be feasible to study one or two electron systems with Z's as high as $\sim 50$. Beam currents the order of 1-5 particle microamps are needed and to have adequate knowledge of particle velocities, the energy resolution of the beam needs to be $\sim 4 \times 10^{-4}$ or better. The vacuum requirements of the beam line for atomic physics are severe because of the large cross sections for electron pickup by highly stripped ions from atoms of the background gas. A vacuum or $10^{-8}$ torr is needed.

\section{Characteristics Required for Materials Science and Solid State Physics}

In ion beam studies of materials near their surfaces, energies of the order of $1 \mathrm{MeV} /$ nucleon will suffice, but for investigating the effect of heavy ions on bulk material properties, energies considerably larger than that figure are required. A nominal energy requirement is $10 \mathrm{MeV} /$ nucleon although for some situations even higher energies would be advantageous. A wide variety of ion species are essential, at least through mass 80 . For damage simulation, currents the order of $1-5$ particle $\mu \mathrm{a} / \mathrm{cm}^{2}$ over areas of $\sim 0.1$ to $\mathrm{cm}^{2}$ are needed. In channeling studies, beam currents lower by 2-3 orders of magnitude will generally suffice, but high energy resolution $\left(2 \times 10^{-4}\right)$ and small angular divergence of the beam $\left(.01^{\circ}\right)$ are required.

\section{E. Ancillary Facilities}

Apart from beam transport, which would normally be considered as an integral part of the accelerator complex, exploitation of any national 
facility will require major capital expenditures for ancillary research instrumentation. Included herein are only the major, fixed, facilities of the type which any potential user might expect to find available for his use in a national facility; further substantial expenditures will be involved in the smaller, more specialized, unique instrumentation which may be required for any specific experiment or group. These latter typically would, however, be funded by the group in question whereas the former would be an essential part of the national facility itself.

These essential ancillary facilities fall into at least three classes. The first includes major groups and associated hardware closely linked to the short and long range operational futures of the accelerator complex. Included here are such items or areas as:

1. Computer complex and group.

2. Hot chemistry and heavy element facilities and groups

3. Ion source development facilities and group

4. Accelerator modernization and development group

5. Target and sample (crystallographic, metallurgical, etc., etc.) preparation facilities and group

6. Biomedical irradiation facility and group
$\$ 2,500,000$

$3,000,000$

500,000

500,000

500,000

$2,000,000$

The second includes more specifically experimental instrumentation of the kind which would typically be installed on a given beam line at a given research station. Included here are such items as:

1. A high resolution magnetic spectrograph $\left(\mathrm{e} . \mathrm{g} \cdot \mathrm{QD}^{3}\right)$

2. A precision goniometer for gamma ray 75,000 correlation studies

3. A high luminosity conversion electron spectrometer 
4. A precision scattering chamber for studies

utilizing semiconductor detectors

(several would be required)

(4@ \$75,000, equipped)

5. A large total absorption NaI(TI) gamma

65,000

radiation spectrometer with anti-

coincidence shield annuli

6. An isotope separator for on-line study of

350,000 short-lived isotopes

7. A high resolution beam pulsing and neutron time of flight facility

150,000

8. A complement of high resolution, large

50,000 volume GeLi or intrinsic Ge gamma ray spectrometers

9. A complement of modular electronics for

200,000 general experimental utilization

10. A recoil mass spectrometer specifically 150,000 designed for very heavy element detection under extreme background conditions

11. A large kinematically overdetermined complex of position sensitive semiconductor detectors

12. An ultra vacuum goniometer system for 50,000 channeling and similar solid state and surface physics studies

13. A large solid angle magnetic spectrometer 50,000 for use in particle-gamma and particleparticle coincidence correlation studies

14. Small stand-alone computers of the process 120,000 control variety (e.g. PDPll and PDP15) which would be dedicated to given major experiments during their effective life

. as active interfaces with the central laboratory computer complex $(4 @ \$ 30,000)$ 
15. An optical spectrograph complex including $\$ 30,000$ quartz optics etc. for atomic physics studies

16. A Wien filter for atomic beam studies

17. A sector magnetic beta ray spectrograph

20,000

The third class includes the major institutional backup facilities that are required for exploitation of an accelerator complex but which would be extremely, if indeed not prohibitively, expensive to develop solely for use with the accelerator. Included are such as:

1. Health physics monitoring and control groups

2. Security and plant protection groups

3. Instrumentation development and repair groups .

4. Fiscal control, purchasing, personnel, etc. groups

5. Hospital and medical groups.

In the first category in an existing national laboratory complex only relatively minor incremental costs would be involved beyond already existing laboratory components. The numbers quoted herein are, at best, somewhat educated guesses for what the costs might be assuming an ab initio start "in the tundra".

In the second category, while no claim to precision in cost estimation is implied, the numbers given are reasonable estimates in 1973 dollars. These items would, with few and obvious specialized exceptions reflecting the eventual site selection, be required for any national heavy ion laboratory.

No attempt has been made to estimate costs associated with the items in the third category since they would be extremely variable depending upon the eventual site of the facility, their partial availability in the neighborhood of the facility and the like. Suffice it to say, however, that in any national facility their availability either as existing components of an ongoing laboratory or as parts of the new facility installation must be assured and, if the latter, they must be included in any facility cost estimate. 


\section{TECHNICAL ALTERNATIVES AND COSTS}

\section{A. Introduction}

Heavy ion accelerators typically consist of an ion source, a first stage acceleration system, a second ionizer for increasing the charge of the original ion, and finally a second or higher stage acceleration system . Several options are available for each of these processes leading to a large number of possible complete systems. Specific configurations differ in the degree to which particular beam characteristics are optimized--reflecting design judgments as to the relative importance of various research objectives-and in the degree to which economic considerations are weighted vs.

performance--judgments as to the research value of particular beam features vs. their cost. As an example, one set of design choices might maximi ze beam intensity, while another might allow much better energy resolution. A given accelerator may even be capable of producing beams of both high intensity and high quality, but it likely to be more costly. The objective of this review is to consider the various possible systems and make judgments as to research effectiveness.

The choice of an ion source is substantially governed by the first stage accelerator, which is chosen not only to achieve certain criteria of beam quality but also with regard to its possible use as an independent accelerator. As a somewhat oversimplified example, a positive ion source in a cyclotron would give intense beams of gaseous elements, with very high energies available for the lighter ions; a negative ion source with a large tandem accelerator would tend to produce any element with approximately. equal ease but with less intensity and with lower energies for the light ions as compared with cyclotrons. Though the construction of these two types of ion source is quite different, the basic objective is the same -- to get the maximum beam through the accelerator for all types of ions. In the positive ion source one tries to produce each ion in a high charge state. For the negative ion system, the beam is accelerated to the high voltage terminal of the tandem accelerator; at that point the beam of ions is stripped to a high charge by passing it through a gas or a thin foil. The resulting positive beam is accelerated further just as in the positive ion source system. Since the effectiveness of the stripping increases rapidly with ion energy, the energy of such a d.c. injector is chosen to be as high as is conveniently available.

Following the first stage accelerator, the ion beam is stripped to a still higher charge state. This stripping process allows more efficient acceleration of the beam, because the higher the charge, the more rapid is the gain in energy in a given accelerating field. However, since there is a significant 
reduction in beam intensity at each stripping, the number of stripping points is usually held to two for negative ion injection and one for high intensity positive ion injection.

Even when the first stage accelerator is capable of accelerating a d.c. ion beam, a following if accelerator such as a cyclotron or linac can only accelerate ions that arrive at the proper phase of the rf cycle. Thus, to achieve optimum performance the time structure of the injected beam must be matched to that of the rf accelerator; this involves the production of short, intense bursts of incident ions at the rf frequency. Some of this beam preparation occurs at the ion source (bunching), but usually some modification of the beam pulse must also take place following the first stage.

Two types of second stage accelerator have been considered, namely an isochronous cyclotron and a linear accelerator. In each of these devices the ions are further accelerated by an electromagnetic wave. The linac consists of a straight line array of radio frequency cavities or circuits; each ion makes a single pass through this array. At each point in the accelerator the increasing velocity of the ion must be matched to a corresponding increase in the velocity of the electromagnetic wave on which it rides. This matching of the velocities is accomplished by varying the dimensions of the linac cavities along the length of the accelerator or by shifting the relative phase at discrete points. A key parameter that is useful in comparing linac designs is the voltage gradient; the rate of increase of the ion energy per unit length of accelerator is:

$$
\Delta E=\text { (charge of the ion) } x \text { (voltage gradient) } .
$$

The acceleration process in a linear accelerator causes the beam to be "radially defocussed," which means that the cross sectional area of the beam perpendicular to its direction of motion will grow as the beam is accelerated; to avoid this, additional focusing elements (usually magnetic quadrupole lenses) are required to give a beam spot of small size.

In contrast to the one pass acceleration in a linac, the cyclotron uses some small number ( 2 or 4 ) of accelerating cavities (dee gaps), but the beam is bent into a circular orbit by a d.c. magnetic field so that it passes through these same cavities over and over again. The increasing velocity of the ion causes it to move in a larger and larger circular path in the magnetic field -proper field shaping brings the ions to the next accelerating gap with just the right phase to be accelerated again, while at the same time providing "focussing forces" which hold the particles compactly together. Acceleration of ions with different mass and charge required changing the strength of the magnetic field and the accelerating frequency. The relation of the maximum magnetic field to the final ion energy is: 


$$
\text { Energy/amu }=k \times\left(\frac{\text { ion charge number }}{\text { atomic mass number }}\right)^{2}
$$

where $k=$ proton final energy (non-relativistic).

The focusing of the ion beam during acceleration is accomplished by introducing "sectors" which are alternate strong and weak regions in the magnetic field.

In the following pages we briefly indicate the state of the technology that is related to the choice of a heavy ion accelerator system. We note there is no clear choice which is superior in all respects; each design has some area of superiority and compromises are necessary; the purpose of this section is to explore the implications of these choices.

\section{B. Large Tandem Accelerator}

During recent years much development work has been done on electrostatic accelerators and several significant improvements have been made in acceleration tube design and in the charging and voltage distribution systems. The High Voltage Engineering Corp. has successfully operated an upgraded MP tandem accelerator at a terminal voltage in excess of $14 \mathrm{MV}$, and very recently, under far from optimum conditions, their TU tandem has achieved $16 \mathrm{MV}$ operation. The National Electrostatic Corp. has demonstrated that their 14UD Pellatron accelerator can very readily attain its design terminal potential of $14 \mathrm{MV}$. In fact, 20 to $30 \mathrm{MV}$ tandem accelerators now appear feasible, and are offered commercially at $20 \mathrm{MV}$.

The advantages of the large tandem accelerator are impressive. The ion beam is of excellent quality and has.very low energy spread, the energy can be varied continuously and,with the exception of a few noble gases,practically all ions in the periodic table can be accelerated. It also appears that the negative ion output of the new sputter-type ion sources is such that, for many ions, the final intensity will be determined by accelerator loading rather than source output. The availability of intense negative ion beams will also greatly facilitate pulsing for time-of-flight studies.

The extent to which a large tandem accelerator will satisfy current scientific interests is difficult to assess. However, it is clear that to exceed the Coulomb barrier for all possible combinations of targets and projectiles would require at least a $40 \mathrm{MV}$ tandem, and that is far beyond the present state of the art. On the other hand, a $25 \mathrm{MV}$ accelerator is thought to be feasible by commercial fabricators; this would enable one to overcome the Coulomb barrier for beams as heavy as bromine on a uranium target. With some loss in 
intensity the projectile mass could be extended up to barium by employing a second intermediate stripper. It is not unreasonable to conclude that such a tandem accelerator could fulfill approximately half of current nuclear and atomic physics requirements in heavy ion physics .

Finally, it is worth emphasizing that the final energy available from a 20-30 MV tandem can be augmented in several ways. One possibility is to use a 10 to $15 \mathrm{MV}$ negative-ion injector -- the latter could either be a new or a modified existing accelerator. A second possibility would be the use of double negative ions (existence of such ions has been established although their life-times have not yet been shown to be sufficiently long to permit acceleration.) A third possibility is to use a post booster accelerator such as a linac or a cyclotron.

\section{Tandem-Cyclotron Systems}

Given a tandem accelerator appropriate for acceleration of heavy ions, its energy range can be extended at relatively low cost by addition of a following accelerator or "after-burner". Groups at Brookhaven, Indiana and Oak Ridge have suggested an open-sector cyclotron as the appropriate afterburner. It is clear that the basic technical feasibility of such an arrangement is solidly established; all major features are reasonable extrapolations from technical systems presently in successful use in various laboratories.

Considering the important question of beam quality, it is likewise clear that techniques for obtaining extremely high quality beams from cyclotrons are solidly established. At low beam currents ( 10 particle nano-amperes) one can anticipate fractional energy spreads of less than $10^{-3}$ and emittance of a few millimeter-milliradians corresponding to the forefront of present cyclotron technology. At higher currents (1000 pna) problems arise and new technical features such as highly efficient bunchers, and a flat-topped rf wave have been suggested in order to obtain intense beams concurrently with high quality. Design calculations on such systems have thus far neglected or only crudely approximated space charge forces; these forces will clearly be a dominant limiting phenomenon and much more accurate calculations are needed both to optimize the accelerator system and to firmly establish beam quality at high current.

Whether the cyclotrons described in the proposals are the best possible cyclotron type after-burner is less clear. All three of the above-mentioned proposed cyclotrons are in fact identical or very similar to structures appropriate for proton acceleration. There is fundamentally much more freedom in designing a heavy-ion cyclotron than in designing a proton cyclotron; several 
structures which would not work for protons are promising for heavy ions -- it seems desirable and prudent to undertake modest explorations of such alternate systems before proceeding with construction of a major cyclotron facility. One such exploration is already in progress at Chalk River (Canada). The Chalk River group is studying an ultra-high-field low-flutter cyclotron in which the main bending field would be produced by a large superconducting coil and the flutter would come from superimposed iron wedges. The Chalk River group estimates that a cyclotron of this type. would be much less expensive ( $50 \%)$ than cyclotrons of the separated sector type. Another possible heavy-ion cyclotron design would use a low-flutter conventional magnet, such as, for example, an enlarged version of the Julich (West Germany) cyclotron. The lowflutter alternatives do involve a performance difference for light ions $(\mathrm{C}, \mathrm{O}, \mathrm{Ne})$; the Chalk River design would be limited to about $30 \mathrm{MeV} /$ nucleon in this region, and the Julich design to about $50 \mathrm{MeV} /$ nucleon, whereas the Oak Ridge design would give $100 \mathrm{MeV} /$ nucleon for such ions. These energies are in every case much higher than the reaction thre shold but, nevertheless, the extra capability of the high flutter machines in this region is an additional distinct research capability which must be weighed against the extra cost.

The subsections which follow consider those three basic cyclotron designs, namely, separated sector (Brookhaven, Indiana and Oak Ridge), superconducting coil (Chalk River) and low-flutter conventional (Julich). For convenience, the designs will often also be referred to as "Indiana type", "Chalk River type" and "Julich type". Major parameters of the three Indiana type cyclotrons and of the Chalk River and Julich cyclotrons are given in Table 5. The discussion in the subsections which follow will assume familiarity with this table or an occasional reference back to the table.

\section{Magnet}

In a real sense the magnet is the fundamental component of a cyclotron. It is highly permanent--once built, it is difficult to change--its characteristics and quality dominantly determine the particle trajectories and fix the quality of the accelerated beams .

A major characteristic magnet parameter of an isochronous* cyclotron is the "flutter"***. The isochronism requirement leads to a smallest possible

* The term "isochronous" implies that the ion orbit time is independent of the energy; this property is necessary to ensure proper acceleration at the dee gaps.

** Loosely "flutter" is the ratio of the amplitude of the azimuthal variation of the magnetic field to the azimuthal average . 
TABLE 5

I. U

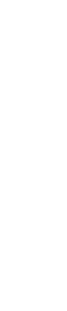

$\Xi$

\begin{abstract}
$\frac{E}{\bar{A}}=k\left(\frac{z}{A}\right)^{2}$
\end{abstract}
225

35

28
0.72

$6: 23$

$\frac{21 \times 37}{72} \simeq 10$

dee volts $(k V)$

$1973 \mathrm{M} \$ / \mathrm{cyc}$.

$\mathrm{Fe}$ (tons)
250

$$
\text { 4. } 5^{*}
$$

2000
BNL

170

0.8

35

20

$5-20$

5.8

250

5.9

830
Julich

ORNL

Chalk River

40

508

1.3

0.96

5.0

30

14.0

45

20

6.0

22

$3-6$

2-9

$2-4$

$9-19$

$7-27$

45

250

$100 \mathrm{kv}$

$\$ 6.14$

$\$ 2.7$

700

2300

300 
flutter, and this smallest value increases approximately linearly with particle velocity. The Brookhaven, Indiana, and Oak Ridge proposals are all so-called high-flutter designs, that is, much more flutter is produced by the structure than is needed for heavy ions. The flutter in these designs is in fact adequate up to energies of 300 to $500 \mathrm{MeV}$ per nucleon. For low particle velocities such as the range 10 to $25 \mathrm{MeV}$ per nucleon, a much lower flutter could be used as in the Julich cyclotron or the superconducting coil design at Chalk River. The advantage of the high flutter separated sector design is the inherent capability of later being adapted for light ion acceleration, and in the fact that there is more room for the rf accelerating system and for the injection system. The principal advantage of a low flutter magnet, whether superconducting or conventional, is that the magnet is much less expensive with fewer alignment difficulties and better linearity. Table 5 gives an indication of the economic advantage of the low flutter magnet. The Indiana cyclotron, for example, uses 2000 tons of iron and, to within $10 \%$, its bending capability is the same as that produced by the 700 tons of iron in the Julich cyclotron. In these large cyclotrons, the iron is the single most expensive item, typically accounting for about $40 \%$ of the cyclotron costs. With either structure it is possible to improve the design and save iron; the Oak Ridge design, for example, uses only $15 \%$ more iron than the Indiana design, but they expect this to give them a $100 \%$ increase in B as compared with Indiana. Nevertheless, there is a basic physical distinction between low flutter and high flutter magnets, and this difference acts to make a low flutter magnet less expensive. Hence, if the designs which have been comparably optimized are compared, the low flutter magnet should always end up as the less expensive choice.

Given that a high flutter magnet is more expensive, the question remains: Are the advantages of this structure (potential light ion capability, easier rf system and easier injection) adequate to justify the higher magnet cost. It is then necessary because of the complicated interactions between components to compare complete designs. Present evidence seems to indicate that the $\mathrm{rf}$ and injection advantages of the separated sector design are relatively insignificant or at least that both an adequate rf and adequate injection can readily be achieved in a low flutter design (see following sections).

\section{Radiofrequency Systems}

The spaces between the magnet of a separated sector cyclotron are relatively free, and designers take advantage of this space by providing an rf system with larger voltage holding clearances and higher $Q$.* With the high $Q$, rf amplifier systems comparable to those on present cyclotrons should

* The quantity $Q$ is a standard measurement of rf system efficiency -- high $Q$ implies greater acceleration for given power input. 
produce around 250 kilovolts per dee, which is about 3 times higher than the $70 \mathrm{KV}$ typical of present cyclotrons: This high energy gain lowers the number of turns and this improves operation in a number of ways, namely (a) vacuum losses are reduced since the number of attenuation lengths is proportional to the number of turns, (b) the final energy will be more precise, (c) rf flattopping is easier, and (d) the separation of turns both at injection and extraction is increased, making both of these processes less critically dependent on exact alignment of the beam.

Low. flutter cyclotrons such as Julich and Chalk River have less room for rf than the separated sector machines, and low flutter machines therefore imply lower dee voltage. Lower voltages can, however, be offset by increasing the number of electrodes; at Julich there are six accelerating gaps and the Chalk River design proposes eight. There is one obvious advantage: if the number of electrodes is doubled and the voltage on each is reduced to half, then the energy gain per turn remains the same, but the total rf power is halved. The separated sector designs could also, of course, use this approach -- with an if system in every valley they could have either double the energy gain per turn for double the power or the same energy gain per turn for half the power. Separated sector designs, however, use the open valleys for both injection and extraction systems. For the low flutter designs, injection and extraction details have, to a considerable degree, not been worked out, and this is an important missing factor clouding a careful comparison. In the meantime, one can note that essentially all present cyclotrons use at least $180^{\circ}$ of the periphery for rf structures, and yet, in all of these circumstances, it has been possible to work out an effective extraction system. It is also basically easier to extract heavy ions because of their low velocity and a consequent enhanced effectiveness of electric fields. Certainly for the low flutter machines it then seems clear that the acceleration advantages of having an electrode in every valley outweigh the disadvantages of the more restricted extraction systems. This might well also be true for separated sector machines -- the questions seem not to have been explored in the present design studies.

Another important characteristic of the rf system is the harmonic number (the ratio of the $\mathrm{rf}$ frequency to the ion orbital frequency). It is the product of harmonic number and the turn number which affects magnet tolerances and energy precision; thus, a low harmonic number is in this regard as valuable as a low number of turns. The rotation frequency for heavy ions (1-2 Mhz) is, however, low compared to values typical in present cyclotrons (10-20 Mhz) and a low harmonic number, therefore, requires operation at relatively low rf frequencies ( $2 \mathrm{Mhz}$ ). Such a frequency. should be good as regards power consumption, for one usually finds that working rf systems in present cyclotrons use less power, approximately linearly as the frequency is lowered. Spiraling of the resonator line as done in linacs would give a compact structure in the right frequency range, but some clever innovation comparable to the distinction between helical and spiral linac structures is needed to obtain 
an adequately rigid and tunable structure. Even without such a breakthrough, one can make important gains in lowering the harmonic number. Looking at Table 1, one notes that the Oak Ridge separated sector proposal plans to. work on harmonic numbers three times lower than the corresponding numbers for the Indiana separated sector cyclotron.

Another important rf question concerns the possible flat-topping of the rf wave by addition of a higher frequency set of rf electrodes running at either twice or three times the frequency of the main rf system. This obvious "good idea" is almost as old as the basic cyclotron concept itself, yet it has never been successfully used in an operating cyclotron, due to severe technical difficulties involved in its implementation. One such difficulty is the phasing requirement. The two rf systems work against each other, one decelerating while the other is accelerating and, if they slide slightly out of an exact phase lock relationship, say by $0.1-0.2^{\circ}$, a difference error occurs which completely nullifies the benefit of the flat-topping. Maintaining such accurate phase locking in a real rf system with significant beam coupling and very different. characteristic structures is a severe technical problem which has, in the past, been beyond the technological limits, but which now seems just at the limits of feasibility. Similarly flat-topping the rf also necessitates 10 times greater accuracy in magnetic field trimming, which seems just at the limits of present technology. The flat-top is, however, not essential to the basic goals of any of the projects and the present state of the several technologies make it appropriate for a serious attempt. It should also be noted that an optional approach to flat-topping is the development of highly effective beam bunchers.

\section{Injection}

The three separated sector cyclotron proposals all include combinations of magnetic and electrostatic bending elements located in the central core of the cyclotron and designed to feed an injected beam smoothly onto a well centered accelerating orbit. In contrast, the Chalk River group plans a stripping injection system similar to the systems presently in use at Orsay, France and Dubna,USSR.

Electromagnetic injection systems offer more flexible performance than a stripping system since there is no constraint on the minimum rigidity of the injected beam,but have the disadvantage of involving a large number of relatively cramped complicated elements--nine independent magnets, for example, in the Oak Ridge proposal--and the system must fit into a relatively cramped central region and be adjustable in position. Electrostatic elements appear substantially more attractive (at $0.5 \mathrm{MeV}$ per nucleon a $100 \mathrm{KV} \mathrm{cm}$ electric field has the same bending capability as a 10 kilogauss magnetic field) and the Brookhaven and Indiana injection systems make considerable use of such electrostatic elements. 
The main disadvantage of a stripping foil injection system is the mechanical problem of providing an adequate foil changing system inside the cyclotron. Foil changing systems are in use at Orsay and Dubna and behave well, but the se machines have substantially lower beams than are expected in the next generation of heavy ion accelerators. The Argonne presentation to the Panel indicated that heating stripping foils to an optimum temperature greatly increased their lifetime. Weighing the various advantages and disadvantages it seems, in summary, that the stripping injection system as used at Orsay and Dubna, and as planned at Chalk River, is a reliable system and would be substantially less costly than electromagnetic injection systems and also easier to use.

In making this suggestion, we do note that stripping foil injection systems place a limit on the energy gain factor of the cyclotron. This arises from the fact that the injected beam must penetrate into the accelerator and smoothly join its appropriate centered orbit. In the case of the Oak Ridge design, this would then limit the maximum energy chlorine ions to about $45 \mathrm{MeV}$ per nucleon, which is substantially less than the limiting energy of the cyclotron magnet proper. Referring to Table 1, we note that the Oak Ridge proposal is the only one with an energy gain factor in the range where stripping injection would curtail the performance range and, even there, the curtailment would only appear as a. moderate reduction in the maximum energy of the lighter ions.

\section{Extraction}

In the separated sector machines extraction is easy because the low average field and the high energy gain per turn lead to a large orbit separation which can be threaded into an electrostatic deflector without manipulation. The extraction problem posed by the ultra-high-field Chalk River design is much more difficult, and a detailed solution to this problem is the main unresolved point in establishing the overall feasibility of such a system.

The low flutter conventional type cyclotron is an intermediate case. Extraction would be more difficult than in separated sector cyclotrons, easier than in the Chalk River structure and approximately comparable to that in most present cyclotrons.

\section{Beam Quality}

The term beam quality is a loosely used expression referring to the capability for doing precision experiments. The characteristics which contribute to the quality are the energy spread and the emittance. Which of these quantities is most important depends on details of the particular experiment to be performed, and hence the vagueness in the definition of quality. 
The dominant final quality parameter in nearly all experiments is energy resolution. In most high precision work, energy resolution is, however, determined by spectrograph magnets, and in these devices energy spreads are converted to spatial spreads and the ultimate resolving power is dominated by the incident beam emittance rather than by the incident beam energy spread. Many factors contribute to the final beam quality of a tandem cyclotron system - the tandem factors are assumed to be well known and the discussion here is restricted to cyclotron factors.

First of all, there is the injection process. Either outside or inside the cyclotron, further stripping is needed to increase the $Q / A$ of the ion. Foil strippers give higher charges and are therefore preferred over gases. They also, however, change the angular spread of the beam by multiple scattering, and this affects the emittance. Since the multiple scattering adds in quadrature to the original angular spread of the beam, its additive affect is greatly reduced if the beam is set up so its divergence at the stripping foil is large compared to the multiple scattering mean angular width. A large divergence means, however, a small spot and the local stress on the stripping foil is therefore increased. Again, however, the developmental work of the Argonne group on heated foils appears to establish that foils can withstand the stresses imposed by a small spot and that the total increase in emittance produced by the multiple scattering can be held to levels of about $30 \%$. This is to be compared with the $100 \%$ increase estimated in the Oak Ridge proposal when a large beam spot is used. There is some question as to whether the small spot technique can be used effectively when the stripping foil is located inside the cyclotron; approximate ion-optics calculations give a favorable indication but should be refined.

A second possibility for lowering beam quality is in the acceleration process itself. The cyclotron magnet and rf system and space charge effects can all act to reduce effective beam quality. Of these, the interaction with the rf system is the least complicated -- if the accelerating voltage is the usual sine wave, the energy gain depends on the rf phase at which the particle crosses the accelerating gap, and the energy spread for a given phase is smallest if the group sits exactly at the top of the rf wave. It is, therefore, important that the control system provide an easy way of insuring that the beam is at the top of the wave. If the if voltage is flat-topped, the requirement is that the beam remain continuously on the top of the wave, and this implies that the isochronism of the magnet has to be about 10 times greater than is required for sine wave acceleration. This goes considerably beyond the accuracy achieved in any presently operating cyclotron but nevertheless seems possible to achieve assuming that advances in magnet measuring technology are combined with very careful design work. 
An energy spread somewhat similar to the spread resulting from the rf phase width comes from the so-called longitudinal space charge effect: particles at the back of the wave are slowed because of the space charge force exerted by particles at the front,and conversely. This phenomenon is one of the most complicated and least understood aspects of an isochronous cyclotron. Present "best" calculations give no more than factor of 2 agreement with experimental measurements. One can, however, qualitatively estimate the effect by scaling from existing machines. Applying this procedure to the Oak Ridge proposal, one sees that the longitudinal space charge limit is near the level at: which the machine hopes to operate. The scaling laws also imply that the energy gain per turn is of overriding importance and if, for example, a Julich type cyclotron were constructed for heavy ion use, it would be necessary to have a much higher voltage rf system to obtain single turn extraction* of microamp beams. On the other hand the four 100 kilovolt dees which the Chalk River group proposes is adequate up to about the microamp level.

The phenomena discussed thus far give direct energy spreads in the accelerated beam. If this spread is small enough so that the particles all make the same number of revolutions in the cyclotron and come out as a single turn, the energy spread can be compensated in a following energy-loss spectrometer. Thus, for a major class of experiments (those studying individual quantum states of final nuclei), these energy spreads can be effectively ignored or considered as not contributing to beam quality. If more than one turn is extracted, the spatial correlation is lost and the situation is then as if an uncorrelated beam with the full cyclotron energy spread were fed to the spectrograph. The cyclotron energy spread would, in this circumstance, be the dominant factor in the experimental resolution and the effective beam quality would be greatly reduced. Single turn extraction is then a dominant factor in beam quality. When it exists, the cyclotron energy spread can be ignored in energy-loss spectrograph experiments; when it does not exist, the full cyclotron energy spread contributes to the resolution.

Achieving single turn extraction is a matter of fulfilling a well established set of requirements on phase width, rf stability and magnet stability. The phase width requirement can, in principle, be achieved with either an appropriate buncher or with a phase selection system. Phase selection techniques are established and in use on several present cyclotrons, and general features of such systems are known. The question of pre-bunching of the tandem beam to

* Single turn extraction refers to an operating condition in which all ions make the same number of revolutions in the cyclotron. This condition leads to greatly improved emittance and hence much better energy resolution in spectrograph experiments. 
meet the phase width goal has been explored only in simplified calculations which neglect many important effects. At average currents of 1 particle microampere, space charge forces are clearly important, and more realistic calculations of these forces are certainly needed before the real effectiveness of bunchers can be reliably stated. Some evidence can be inferred from present tandem Van de Graaff bunchers, although the target time pickoffs generally used with such systems are insensitive to many drifts of importance in injecting phasing.

Another type of beam quality deterioration comes from time changes in the magnetic field of the cyclotron. These changes move the beam and therefore increase the emittance presented to static devices. Field changes also shift the rf phase and, therefore, change the energy and break the correlation between energy and position. The result in the extreme is a larger emittance and an uncorrelated energy spread which cannot be sorted out in an energy loss.spectrograph. This, then, means that precise regulation of the magnetic field is extremely important. Goals should certainly be at the level of one part in 100,000. This is possible with present technology provided the requirement is clearly noted as magnet power supplies are being designed. It is also possible to arrange the rf phase in a way which minimizes the energy change produced by a given set of magnet fluctuations; this technique should be used.

Other contributions to the beam quality can come from time fluctuations in any machine element (inflectors, deflectors, etc.) from a variety of coupling phenomena (the most important of which would appear to be from the rf flat-topping cavities), from radial variation of the rf wave form and, finally, from phenomena such as multiple scattering in the residual vacuum. All of these phenomena appear relatively less important than factors already discussed, and it is therefore reasonable to pass over them in this review.

In summary, it seems that the quality goals indicated in the proposals are clearly feasible. If the Argonne foil heating technique can be successfully employed, one could even expect significantly better quality than anticipated in the Oak Ridge proposal.

\section{Cyclotron-Cyclotron System}

The French heavy ion study group (GANIL) proposes a primary accelerator system consisting of two large cyclotrons, each with an energy 
parameter (" $\mathrm{K}$ ") of $380 \mathrm{MeV} . *$ After the first cyclotron, the beam is stripped to a higher charge state and then injected into the second $\mathrm{K}=380$ machine. At this point, the rf bunches in the output beam from the first cyclotron must be matched to the acceleration system of the second cyclotron; this imposes difficult constraints which must be fulfilled. The difficulty comes from the fact that the orbital frequency is proportional to the ion charge $(\omega=Q B / M)$ and when the charge is increased in a stripping process, the orbital frequency is also increased in the same proportion (assuming the magnetic field is the same in the two cyclotrons). Dealing with this change in the orbital frequency is awkward. Three main techniques have been suggested, namely:

a) Ignore the pulsed nature of the beam from the first cyclotron and run the second cyclotron at the natural frequency which corresponds to the most abundant charge state of the stripped beam. In this case, the capture efficiency in the second cyclotron would be as if a DC beam were injected, namely about $2 \%$ without a flat top and $10 \%$ with a flat top, and hence a large loss in current results.

b) To avoid the large capture loss which follows from asynchronous injection, the rf systems in the two cyclotrons can be operated synchronously either at the same frequency or at some rational multiple. The ion rotational frequency in the two cyclotrons must then, however, also be related by the same combination of integers as the rf systems, which means that only particular ion charge states can be allowed (or, alternatively, the magnetic field in one of the cyclotrons must be reduced from its peak value, thereby reducing the maximum energy of the system). The French, for example, design on the basis of an ion orbital frequency ratio of 4 , i.e., the orbital frequency in the second cyclotron is always exactly four times greater than the frequency in the first cyclotron. Table 6 shows the impact of this choice for uranium ions. The charge state which satisfies the energy relation is five units higher than the most probable charge, and the intensity in this charge state is only about $40 \%$ of the intensity in the peak charge state. The French design also elects to use the same harmonic number in the two cyclotrons, which means that the rf frequency in the second cyclotron is four times higher than the frequency in the first. This means, however, that only $1 / 4$ of the possible acceptance windows in the second cyclotron actually receive particles, and there is then a $75 \%$ reduction in intensity. This, together with the required selection of an ion off of the peak charge, gives a total reduction of about a factor of 10 and the system performance is, therefore, essentially the same as would be obtained from asynchronous coupling.

* The French proposal is, in fact, really a three stage cyclotron system, in that the injector into the first of the large cyclotrons is itself a small cyclotron. No stripping would be used between the injector and the first large cyclotron, however, so the effect of the se two elements is the same as a single $\mathrm{K}=380$ cyclotron starting from 0 energy. 


\section{TABLE 6}

\begin{tabular}{lcccc}
\hline $\begin{array}{c}\text { Ion } \\
\text { from } \\
\text { source }\end{array}$ & $\begin{array}{c}E / A \\
\text { after 1st } \\
\mathrm{K}=380 \\
\text { cyc. }\end{array}$ & $\begin{array}{c}\text { most } \\
\text { probable } \\
\text { charge }\end{array}$ & $\begin{array}{c}\text { required } \\
\text { charge } \\
\text { for 2nd } \\
\text { cyc. }\end{array}$ & $\begin{array}{c}\mathrm{E} / \mathrm{A} \\
\text { after } \\
\text { 2nd } \\
\mathrm{K}=380 \mathrm{cyc} .\end{array}$ \\
\hline$U^{7+}$ & 0.33 & 25 & 28 & 5.3 \\
$U^{8+}$ & 0.43 & 28 & 32 & 6.9 \\
$U^{9+}$ & 0.545 & 31 & 36 & 8.7 \\
\hline
\end{tabular}

c) A third option is to run the two cyclotrons at the same frequency and change the harmonic number. The French system, for example, uses harmonics $2,4,6$ and 8 in both cyclotrons. If both cyclotrons were operated at the frequency proposed for the second cyclotron, the harmonics in the first cyclotron would then be $8,16,24$ and 32 and every rf bunch in both machines would be filled. Again, however, there are difficulties. The dee form factors are dependent on harmonic numbers and the $30^{\circ}$ dees which the French propose would give no acceleration on the 24th harmonic and would have large transit time corrections on the other harmonics. Reducing the dee angle in the first cyclotron by the same factor of four as the harmonic number (to $7.5^{\circ}$ ) would make the form factor the same for the dees in the two machines, but would give very large transit time corrections. High harmonics are also bad since they make precise operation much more difficult (see discussion in the tandemcyclotron section).

As of this time, no good solution to the two cyclotron matching problem has been presented. Possible improvements for selected ions could be made by individual tailoring of the characteristics of the two cyclotrons. The rotation frequency ratio of four selected by GANIL would, for instance, be much better accomplished for uranium ions if the first cyclotron had an energy parameter of about 600 . This means, however, that the two cyclotrons must be separately designed and significant additional costs would therefore arise. Since the se matching difficulties do not arise in a Tandem-Cyclotron system, the Panel feels, based on the evidence now available, that performance of a tandem-cyclotron system should be superior to that of a two cyclotron system. 


\section{E. Linear Accelerators}

Recent design studies indicate that modern linacs can overcome many of their early limitations in much the same way that cyclotrons have in recent years evolved into precision accelerators. Designs emphasize the need for a high macroscopic duty cycle and the ability to accelerate a wide range of ions. Subdividing the linac into independently phased sections has also been rediscovered; this allows variable energy operation and also gives higher energies for higher $Q / A$ (in contrast with the old fixed velocity characteristic of linacs). The separately phased short sections are possible as a result of the improved phase and amplitude control developed at LAMPF and at the high current injector projects at BNL and NAL. To achieve comparable performance criteria, there are a number of possible designs, each of which offers marginal advantages over the others. In the following sections, the se alternatives are discussed without reference to specific designs given in the proposals.

\section{Superconducting vs. Room Temperature Structures}

In either case it is possible to have a high duty cycle $(\sim 100 \%)$ for reasonable gradients (1-3 MV/m). Thus, the choice must be made on the basis of initial cost, operating cost, reliability and technological difficulty. Primarily due to the large RF power requirements in a room temperature design, a superconducting accelerator will probably have both lower initial cost and lower operating expenses. The question of reliability cannot be answered with any assurance until a superconducting machine has been in use as a research tool. Historically, new devices invariably offer new problems. Possible difficulties of a superconducting linac include: sensitivity to vacuum accidents; degradation of the superconducting surfaces due to radiation damage; instabilities related to the high $Q$ of the structure; instabilities related to mechanical vibrations. Though there are probably solutions to the se problems and no overwhelming obstacles to construction, one should expect a 2-3 year development period before a final design is in hand. (In contrast, approximately one year would be required to arrive at an optimum design for a room temperature device.)

\section{Basic Configuration}

Though linacs can be built to handle particles injected at velocities below $0.01 \mathrm{c}$, most of the new designs choose a higher energy injector in order to give better beam quality. The optimum choice for an injector is not well defined. It is definitely cheaper to choose a relatively low energy injector (especially when compared to a $20 \mathrm{MV}$ terminal tandem); however, the tandem would offer a superior quality beam for many ions when used indepen- 
dently. Based on present ion source and tandem technology, it seems clear that one can get greater beam intensity by the SuperHILAC system of accelerating high charge state positive ions in a small injector and then stripping the ions part of the way along the linac. The double stripping process in the tandemlinac system will mean a beam of lower intensity.

Final beam energy resolution would be comparable with either system, and if the intensity is sufficiently high, either restricting the longitudinal phase of the incident beam or using magnetic analysis, can give beams of high quality $\left(\Delta \sim 10^{-4}\right)$. Debunching for either injection configuration should be considered very seriously.

\section{Accelerating Structures}

The low phase velocities appropriate for heavy ion acceleration require a low rf frequency. Several alternatives exist: cylindrical resonant cavities; helically loaded resonators; and a variety of interdigital drift tube configurations. Most can be adapted to superconducting structures; however, some offer more difficulties in this regard and will be singled out in the following discussion.

a) Cylindrical cavities: versions of the standard TM cavities are used in the SuperHILAC and Unilac to allow energy variation and the accomodation of a wide range of particle masses. For the low frequencies required, these devices have large transverse dimensions and thus are not easily adaptable to superconducting structures. A variant on this is the highly re-entrant cavity which has smaller transverse dimensions. It is, however, doubtful that this configuration can be used below a velocity of $0.05 \mathrm{c}--$ and even there the high frequency ( $400 \mathrm{MHz}$ ) will make beam injection difficult.

b) Helical resonators: the se structures offer both small transverse dimensions and low phase velocities. They are, however, relatively flexible and thus are very sensitive to mechanical instabilities and radiation pressure driven frequency shifts. Overcoming these problems requires an elaborate feedback stabilization and control system. In the room temperature version, the low shunt impedance causes high rf costs, and the high resistance to water flow in the helix requires a high pressure cooling system. In the superconducting version the lack of rigidity becomes a much more serious problem due to the high $Q\left(10^{8}-10^{9}\right)$ and no control system now exists that can handle accelerating fields of greater than $\sim 1.5 \mathrm{MV} / \mathrm{m}--$ though it is thought that this limit can be overcome.

In the cases considered, small groups of $\lambda / 2$ resonators would be phased independently for greater flexibility in accelerating a wide range of masses. 
c) Drift tube structures: Most existing linacs are of this form and are based on a fixed velocity profile along the machine. These designs involve such large transverse dimensions that they are easily excluded for a superconducting accelerator. To lower the frequency, and at the same time obtain a reasonable size, one must consider structures where the resonators are loaded by the stems and drift tubes. Two ideas of this sort have been studied with regard to use in superconducting hilacs: a tapered 1/4 wave stem and a spiral loaded resonator. Superconducting models of the latter have mechanical and electromagnetic stability that is about a factor of 100 better than for helical resonators, and at the same time allow a larger accelerating field before critical magnetic field breakdown is reached.

As in the case of helical resonators, small groups of these resonators may be phased separately to give a variable velocity profile along the accelerator. In general, the spiral resonator offers the same low frequency-low phase velocity properties as the helix; however, at room temperature the shunt impedance of the spiral is about twice that possible for the helical resonator. At the same time, the impedance to water flow is very much lower allowing it to be cooled with a low pressure water system.

\section{Focusing}

In a phase stable linac, a major part of the accelerator cost lies in the magnetic quadrupole lenses that keep the beam from diverging in the radial direction. All the designs considered involve such a configuration. The only alternative to this choice is that of "alternating phase focusing" in which the phase of the particles relative to the rf accelerating field is allowed to vary in such a way that one gets both longitudinal and radial stability -- in direct analogy to the principle of alternating gradient lenses. Though this method would reduce the cost of the accelerator, it can be made to work only by reducing the longitudinal phase space of the injected beam, thus lowering the beam intensity. It remains, however, for future calculations to decide whether there are ways to improve the acceptance of a purely alternating phase focused accelerator or whether a combination of magnetic quadrupoles and alternating phase focusing could be effectively used.

\section{Beam Injection}

Because linacs tend to operate at somewhat higher frequencies than cyclotrons, the preparation of a suitable pulsed beam from the injector poses greater difficulties. It is clear that much work remains to be done in this area because the slowly moving, highly charged heavy ions are more susceptible to space charge effects. If the linac is to be operated only as a 
very high intensity source, present designs are equal to the task; however, raw beam resolution in the $10^{-4}$ range requires much better pulsing systems than are presently available. This comment may also be applied to the cyclotron systems, but there the problem is somewhat less severe due to the lower frequency.

\section{Summary}

By its intrinsic nature, the linac cannot accelerate light ions to as high an energy as a cyclotron if one fixes the energy of the heaviest ion beam to be produced. However, by separately phasing sections of the linac, the light ions can be made much more energetic than in a fixed velocity profile structure. It should be possible to insure that the beam quality for a linac is comparable to that of a modern cyclotron, and at the same time the linac may more easily be extended if need arises. The one problem that seems more difficult for the linac is in beam pulsing.

In comparing room temperature to superconducting linacs, there are still a sufficient number of unknowns that a final judgment is not possible. The initial cost and operating expenses of the superconducting system appear to be lower, but there are still no adequate tests of the long-term reliability of such a device. Considering both this question and the still unsolved (but not insoluble) technological problems that remain, one must expect a 2-3 year development period before a firm design is fixed. A superior room temperature design that involves a variable velocity profile and a large macroscopic duty factor in which one has good raw beam resolution but can also handle high intensity beams would probably require about a year of intensive design study.

It is not clear that the tandem accelerator is a necessary ingredient in either the superconducting or room temperature versions unless its unique properties as a separate facility are required. The insistence on the use of a tandem allows (for the present at least) beams of limited intensity compared to those obtainable from a positive ion source. One should, however, use some sort of pressurized injector in any design to improve the beam quality of the linac -- a $4 \mathrm{MV}$ injector (single ended) is probably the least that one should consider.

\section{F . Upgrading of Existing Facilities}

During the past 7 or 8 years, there has been a marked reduction in the funding of new accelerators and little money has been available for the improvement of existing facilities. It has become clear that there is a need for an upgrading program for some of the existing accelerators . 
The problem we consider here is not the general need for upgrading but rather the feasibility of improving at least one accelerator to the point where it could become an effective heavy ion facility. By "effective" we mean an accelerator capable of accelerating ions from most of the periodic table to energies of at least $7 \mathrm{MeV} /$ nucleon with beam intensities in the range $10^{12}$ to $10^{13}$ particles/sec. The energy definition should be about 1 part in $10^{4}$ and the energy should be continuously and easily variable.

This is an extremely difficult problem to specify because there are a large variety of ways that an accelerator can be upgraded and many schemes that seem attractive have yet to be tested. One promising approach would be to create a hybrid facility using an existing cyclotron and an existing tandem accelerator. Another more expensive approach would be to build an energy booster for one of the existing large tandem accelerators.

A possible choice of a booster cyclotron is the sector cyclotron presently under construction at the University of Indiana. The advantage of this machine is the sector nature of its magnet which, at least in principle, should facilitate injection problems. However, this cyclotron was designed primarily for light ions and the necessity of using high harmonic acceleration for heavy ions is less than ideal. In considering this accelerator's unique capability for light ion acceleration and the delay that would result in its conversion, the nuclear physics community is better served by seeking other candidates for a major heavy ion facility.

The other possibility is to add a booster to one of the largest existing tandem accelerators. Many alternatives are possible: A separated sector cyclotron, a conventional isochronous cyclotron, a superconducting cyclotron, a room temperature linac or a superconducting linac. In all cases, the difficulty encountered arises from the energy limitations of existing tandems which result in low charge to mass ratio of the injected ion beam and hence relatively ineffective utilization of the booster. Thus, the booster must be large and expensive or,alternatively, the energy of some ions will be well below reaction thre shold.

In summary, many existing accelerators could be upgraded to the point of becoming useful heavy ion facilities; the general goals of the national program cannot, however, be met unless the upgrading program is in magnitude comparable to the construction of an Option I or Option II facility. 


\section{SuperHILAC}

The SuperHILAC is designed to accelerate all ions through uranium to an energy of $8.5 \mathrm{MeV}$ per nucleon. There is a great advantage to the scientific community in improving the operation of this existing machine so that its operation is brought to maximum efficiency as rapidly as possible. Although the design capabilities of this machine in terms of projectile energy and beam current (optimistic particle fluxes of $3 \times 10^{14}$, $3 \times 10^{12}$ and $10^{11}$ per second are quoted for ${ }^{16} \mathrm{O},{ }^{84} \mathrm{Kr}$ and ${ }^{238} \mathrm{U}$ ) are suitable for a large number of heavy ion experiments, the ancillary experimental equipment and experimental space are limited. This is understandable in terms of the fact that the SuperHILAC was built at the "bare bones" level by removing the old HILAC from the building and replacing it with the new accelerator. Additional funds necessary to maximize the capacity of the SuperHILAC were estimated at $\$ 1 \times 10^{6}$ at the Panel's meeting in July; this number is probably a minimum estimate.

The SuperHILAC has the capability to produce useful beams of many projectiles across the periodic table at energies above the Coulomb barrier for all targets. Hence, this machine will open up new fields of research in nuclear physics and chemistry and other areas of science. The beam characteristics of the SuperHILAC are such as to exploit the typical features associated with heavy ion reactions; namely, the high nuclear charge of the ions and large transfers of linear and angular momentum for nuclear science research and in attempts to make the superheavy elements . Furthermore, reactions are possible between heavy projectiles and targets to study the collective and macroscopic behavior of nuclear matter.

At present, the questions about the beam quality, ease of energy and beam variation are under study and little is known concerning the influence of operating parameters on the beam characteristics. However, it is commonly felt that the beam characteristics of the SuperHILAC are not sufficiently refined for many classes of experiments which require high energy resolution.

The SuperHILAC uses two injectors. The lower energy injector, a 750 KV Cockcroft-Walton is capable of acceleration of ions up through ${ }^{40} \mathrm{Ar}$. For the acceleration of heavier ions a second injector, a 2.5 MV Dynamitron, is used. This second injector has been very unreliable and only on very few occasions have nanoampere beams of krypton been available for experiments; correction of this situation may require extensive modifications . 


\section{Bevalac}

The Bevalac at the Lawrence Berkeley Laboratory is a combination of two existing machines, the SuperHILAC linear accelerator and the Bevatron synchrotron. Major costs have already been funded; construction of beam lines and upgrading of the machines are underway. The planned time schedule will result in operation of beam lines in one year and completion of the time-sharing pulse selector system in two years. The SuperHILAC alone is capable of producing heavy ions up to $8.5 \mathrm{MeV} / \mathrm{amu}$, with intensities ranging from $10^{15}$ ions/sec for ${ }^{12} \mathrm{C}$ to $10^{11}$ ions/sec for ${ }^{238} \mathrm{U}$. When the se beams are chopped and accelerated in the Bevatron at 10-17 pulses $/ \mathrm{min}$, the energies will be raised to $2.5 \times 10^{3} \mathrm{MeV} / \mathrm{amu}$ at intensities of $10^{10}$ ions/pulse for ${ }^{12} \mathrm{C}$ and $10^{4}$ ions/pulse for ions heavier than ${ }^{56} \mathrm{Fe}$.

\section{3. $\underline{P P A}$}

The Princeton-Pennsylvania Accelerator is a fast cycling AG synchrotron designed to produce $3-\mathrm{GeV}$ protons. Following termination of AEC support it was operated for one year as a heavy-ion accelerator producing ions such as ${ }^{14} \mathrm{~N}$ at energies up to $530 \mathrm{MeV} / \mathrm{amu}$. The accelerator is now shut down.

The PPA could be revived as a heavy ion accelerator of modest capability for ions such as ${ }^{40} \mathrm{~A}$ at energies of $100-400 \mathrm{MeV} / \mathrm{amu}$ and at intensities of $10^{10}$ ions/second. The cost of upgrading and of operation would be $\$ 1.0$ to $\$ 2.0$ million/year. In addition, about twice this amount would be needed to develop a suitable laboratory to exploit the available properties of the heavy ions and to enlist competent staff.

\section{G. User Needs, Manpower Considerations and Operating Costs}

The Panel considered the measures required for the most effective scientific exploitation of heavy ion facilities in light of the needs of the national heavy-ion program. Important data that will be discussed are the number of scientists who can be involved and the corresponding number of scientific man years (SMY), which implies a certain level of funding. The question of how the national program should be organized is answered in part by the Panel's recommendation that the SuperHILAC, as well as the facilities required under Option I or Option II, be national facilities. The considerations which led to this recommendation are summarized in the following.

\section{User Needs}

For these facilities to contribute most effectively to the national heavy-ion program, it is essential to encourage the development of experiments 
of the greatest scientific merit and to give these the highest priority. It is thus necessary to draw on the talents of the entire heavy-ion science community, to select the best experiments from among those they would propose, and to provide the necessary services as well as the technical and scientific assistance at the facilities so that such experiments can be planned and performed expeditiously. These requirements constitute a definition of "national facility."

The use of the facility should be open to all on the basis of the scientific merit of their proposals, without regard to whether the proposers are members of the facility's staff (in-house users) or are nonstaff scientists (outside users) who reside at the facility only during the periods necessary for planning and carrying out their experiments. Toward this end the Panel recommends the appointment of a scientific program committee drawn from experts in heavy-ion science. A majority should be from outside the facility staff. They should be chosen on the basis of their competence and should comprise as broad a representation of heavy-ion science as possible. The membership of the committee should be rotated frequently. Scientists wishing to use the accelerator should submit their proposals to the committee, which would evaluate the scientific merits of each, rank ordering them if possible. Their recommendations should be followed largely by the facility management. The users should be informed of the decision of the program committee and the laboratory management as soon as possible.

It follows that both the funding agencies and laboratory managements must carefully and explicitly plan to satisfy the needs of the outside users . Adequate funding must be provided for the outside users. The laboratory management must meet their needs at the facility for equipment, support personnel, and housing. It should encourage the participation of outside users and be especially sensitive to their needs. The outside users should be informed that the laboratory management assigns a high priority to carrying out meritorious experiments as expeditiously as possible.

The users should know when their experiment is scheduled to be performed and the period of time allocated for it as soon as possible.

The staff of the facility should assist the outside users in the design and planning of experiments. The laboratory should provide the logistic support needed to set up and perform experiments at their scheduled time. Assistance of this kind is most important in assuring effective use of the facility.

Except for expendables such as targets and detectors, equipment communally used--for example, scattering chambers, electronic equipment, 
and computers--should be provided by the facility. The Panel did not discuss how the costs for the se services should be allocated.

Housing for both short (days to weeks) and longer periods (months) of residence at the facility should be provided for outside users.. . Graduate student housing is an important need.

The measures discussed in this section should be implemented at the SuperHILAC and should be planned for any new national facility.

\section{Manpower Considerations}

The principal emphasis in this section is on the scientific manpower required to obtain the most effective use of an Option I type facility.

The machine parameters of Option I will overshadow those of any heavyion accelerator in the nation. Therefore, it will be the forefront accelerator, the most desired accelerator, for a national community numbering more than approximately 600 nuclear and atomic physicists and nuclear chemists whose main interests are to investigate phenomena with heavy ion beams and who represent every geographic area of the country.* (About 150 of the se heavyion scientists are full-time research scientists at national laboratories. Two thirds of the scientists are east of the Mississippi. More than 40 universities have scientists engaged in heavy-ion research.) Insofar as management of a single accelerator can do so, it must be responsive to the national community of heavy-ion scientists, encouraging and meeting the needs of the maximum possible number of users. This is not only a matter of equal opportunity to share unique equipment of broad appeal, which the scientific community believes is critical to the health of nuclear science; it is also a matter of. cooperation and funding. To be effective as a national accelerator, the facility must have adequate funding for both the necessary support facilities and the special needs of outside users who wish to mount independent research efforts at the facility as discussed in the preceding section.

There is wide variation in the estimated number of SMY (scientific man years of effort) that the different proposed facilities are expected to support. Much of the scatter in the numbers arises from differing accounting procedures for graduate students and outside users, though there is also a broad range of

* This number, which is quite approximate, does not include an estimate of the number of solid state and biomedical scientists who will wish to use such a facility. However, their number is thought to be relatively small. 
opinion as to the number of experiments a scientist can complete each year, the length of an average experiment, the number of experimenters per group, and the like.

These subjective considerations can be largely circumvented by basing the estimate on data from existing facilities whose heavy-ion beams are in great demand. These data should provide a reliable base for extrapolation, since the number of scientists that a given accelerator is capable of serving does not depend sensitively on beam parameters such as energy or atomic species. The figures we have collected are modified to take into account expected advances in sophistication and flexibility of the beam handling and data acquisition as well as the possibility of a facility providing more than one simultaneous beam for experiments.

The Brookhaven Double MP Tandem Facility with its conspicuous and actively supported user's program is close to being a prototype for a national heavy-ion facility. This actively used facility supports 35-40 scientific man years per year, about 20 of which are produced by Brookhaven staff. The 15-20 SMY of outside users and collaborators are made up from about 50 scientists and 10 graduate students, most of whom are summer visitors.

The Oak Ridge Isochronous Cyclotron (ORIC) has a scientific staff of 30 who use approximately $70 \%$ of the very tight beam schedule. The 75 scientists from outside Oak Ridge who make use of ORIC each year, often in collaboration with in-house scientists, comprise another 15 to 20 SMY per year.

The Munich MP Tandem Facility supports about 50 SMY per year plus 20 to 25 graduate students. There is no outside user program. This facility is probably the most heavily booked of any heavy ion facility in the world. Most observers consider it to be so overcrowded that the scientific staff is not properly served and turnsto other accelerators for part of their research.

On the basis of experience with existing accelerators, the Option I facility with a single beam would be expected to support about 50 SMY of effort each year. We expect, for several reasons, that the actual number of SMY's will be higher than this.

Option I is projected to be a more sophisticated facility than any of those listed above. New developments in ion source technology and in computer assisted beam handling should reduce turn around times between experiments and should improve the reliability of the accelerator, thus reducing the effective time for a given experiment. Moreover, it is expected that multiparameter acquisition of data will be the rule whenever possible and the 
Panel strongly recommends that data acquisition apparatus be developed to reduce, insofar as is practical, the time for doing a given experiment. The Panel is, however, mindful that the increased sophistication of data acquisition and analysis will not necessarily lead to a commensurate reduction in the average time for doing an experiment since each improvement will undoubtedly be the impetus for scientists to carry out additional aspects of investigations which would otherwise be impractical. Thus the complexity of the experiments will tend to keep pace with new developments in apparatus, thus maintaining the time required for a given experiment about constant.

A substantial gain in the availability of the accelerator for users could come from the development of simultaneous beams of particles whose parameters can be independently varied. No heavy-ion accelerator has such a capability now. Implementation of such beams will require a substantial development effort. Simultaneous experiments of a highly restricted nature are, however, possible with existing technology. It has been proposed, and it should be practical, to make use of the beams of different charge states that emerge from the electrostatic accelerator, sending one of the beams to the cyclotron for further acceleration to a high energy target room, while taking one or more of the other charge-state beams for use in low energy target rooms. The implementation of such state-of-the-art techniques is not expected to increase the effective usefulness of the facility by more than 25 percent, but such a gain is clearly desirable and is recommended,as is the early development of simultaneous beams of differing species and energy.

With the expected improvements in beam handling, machine reliability, data acquisition, and some use of simultaneous beams of differing species and energy, the Panel concludes that the Option I facility will support a scientific effort of about 70 SMY each year.

The balance between the size and strength of the in-house staff and outside users is governed by a number of factors. On the one hand, the facility must serve the national community, which is far too large to be adequately served by a single accelerator. On the other hand, a strong on-site staff is necessary for the continuity of long-range programs, for the procurement, installation, and maintainence of front-line equipment such as spectrometers and scattering chambers, which will be used communally and, not least important, for the collaborative experiments with smaller outside groups from all parts of the nation that are expected to be an important part of the scientific program.

Experiments must be selected on the basis of scientific merit, with both in-house and outside users competing for machine time. Conflicting needs must be resolved in accordance with this criterion. 
It is the Panel's view that the strength, diversity, and needs of the outside community dictate that no less than 50 percent of the available machine time be allocated to outside use. The outside users must be adequately funded to make use of this opportunity. The in-house scientific staff must be strong enough to command at least 25 percent of the machine time. There is no doubt that the attractiveness of the Option I facility will attract a staff that will maintain the standards of independent research as well as the standards for close collaboration and support of outside users that is envisioned for the facility.

The se considerations lead us to conclude that the Option I facility should have about 25 full-time scientists on the staff. The number of outside users during a given year is expected to be no less than 100 and will likely be closer to 150 and be equivalent to from 35 to $50 \mathrm{SMY}$.

In summary, the proposed accelerator is expected to support about 70 SMY of effort in heavy-ion research each year. About 25 to 35 full-time scientists are expected to be staff members of the National Heavy Ion Facility, and more than 100 outside scientists are expected to use the facility in a given year. Even so, the conclusion is inescapable that a single National Heavy Ion Facility will serve but a fraction of the scientific community engaged in heavyion physics. And as the evidence cited above indicates, the estimate of 70 SMY is optimistic for a single-beam facility .

\section{Operating Costs}

The Panel received information indicating that costs in FY 79 dollars for operation of an Option I facility, including expenditures for in-house support of outside user activities, would total about $\$ 3.2 \mathrm{M} /$ year. Using a rough estimate of an additional expenditure of $\$ 85 \mathrm{~K} /$ year per SMY of research yields approximately $\$ 6.0 \mathrm{M}$. The projected total operating and research costs in FY 79 dollars would thus be about $\$ 9.2 \mathrm{M}$ /year. This would correspond to about $\$ 130 \mathrm{~K} / \mathrm{SMY}$ of effort .

\section{H. Costs}

The oral presentations and written submissions to the Panel included substantial information on estimated construction costs of a number of different types of heavy-ion accelerator facilities. The Panel has not carried out independent detailed reviews of these cost estimates but has given consideration to the realism and completeness of them. 
The Panel believes that a heavy ion facility satisfying Option I, consisting of an electrostatic accelerator injecting into an isochronous cyclotron, can be constructed at a cost of about $\$ 30,000,000$. This estimate assumes funding in FY 1975. Further, it assumes construction at an existing major research laboratory to benefit from existing buildings, capital equipment, and supporting services. Under these assumptions, a rough breakdown of construction costs and ancillary equipment costs for an Option I facility is as follows:

\begin{tabular}{lc} 
Accelerator building & $\$ 9.4 \mathrm{M}$ \\
Cyclotron & 7.7 \\
Tandem & 5.6 \\
Beam transport system & 1.8 \\
Computer control systems & 1.0 \\
Radiation safety and moni- & 1.5 \\
toring, quality assurance, & \\
\multicolumn{2}{l}{ and miscellaneous items } \\
Ancillary equipment \\
$\quad$ Total & $\underline{3.0}$
\end{tabular}

As noted in the preceding section, the total operating and research costs for an Option I facility in FY 79 dollars would be about \$9,000,000 per year.

As noted in Section IV, ancillary facilities are a vital component of a national heavy-ion facility and comprise a significant percentage of the total financial investment. The $\$ 30,000,000$ cost estimate for construction of a facility satisfying Option I presupposes the availability of most of the ancillary facilities. If it were necessary to provide additionally the broad research and service facilities, including a computation center, at least an additional $\$ 10,000,000$ would be required. The major experimental instrumentation that would be required to carry out a first-rank multidisciplinary research program is estimated to cost in the range of $\$ 3,000,000$ to $\$ 5,000,000$. Again, a large active research center would already possess much of this instrumentation. The cost of the other ancillary facilities and services, largely administrative in nature and necessary as back-up for the exploitation of an accelerator complex, would be difficult to estimate since it would depend largely upon the eventual site of the facility, their partial availability in the proximity of the facility, and the like. Their availability, however, must be assured as part of any ongoing laboratory or, if as part of a new facility installation, the se required facilities must be included in the total facility cost estimate.

* Assuming funding in FY 75 . 
The Panel recognized the possibility of lower construction costs for other systems such as those employing cryogenic accelerator technology, but the Panel has judged that such systems require further research and development before being adopted and recommends that funds be provided for this. purpose.

The Panel was impressed with the capability offered by the higher voltage electrostatic tandem accelerators in the 20-25 MV range for heavy-ion research and has discussed the facilities under Option II. However, it must be emphasized that the capability for heavy-ion science under this option would fall far short of that offered by Option I. The Panel has reviewed the major components of cost estimates for a $25 \mathrm{MV}$ facility, again located at a major, active research center, and is satisfied such a facility can be established for about $\$ 14,000,000$. A reduction in costs of perhaps $\$ 3,000,000$ would result if a lower terminal voltage of about $20 \mathrm{MV}$ was accepted, but there would be a loss in the nuclear research range through the periodic table.

The Panel received information from the Lawrence Berkeley Laboratory as to the status of the SuperHILAC including its operational capabilities and the extent of the accommodation of user groups in the context of operating as a national heavy-ion facility. The Panel has not evaluated a detailed cost estimate for the upgrading of the SuperHILAC but strongly urges that funds be provided to insure operation of the accelerator to a reliability level that would permit at least 16 eight-hour shifts of useful research time. The Panel, in particular, notes the need for improvement of the $2.5 \mathrm{MV}$ injector, improvement of the rf system, computer control, and additional experimental area and service support for user groups. The Panel believes that funds in the range of $\$ 2,000,000$ to $\$ 3,000,000$ should be provided for this purpose. Operational support for the SuperHILAC should be such as to permit the facility to serve as a national facility available to all on the basis of the scientific merit of their proposals.

The Panel feels that the present broad-based program consisting of several modest heavy-ion accelerator facilities should be maintained and that these facilities should continue to be upgraded. Some of the major facilities. (at Rochester, Brookhaven and Yale) are already in the process of being upgraded to increased heavy ion performance. It is important that increased operational support be made available so that this increased research potential can be supported.

The high-energy heavy-ion research that will become possible with the operation of the Bevalac has been considered. No further capital improvement of this facility beyond the current construction project has been proposed by 
Lawrence Berkeley Laboratory. The Panel has been informed that the BevatronBevalac will be supported from several budgets and has already commented on primary justification for support of this facility.

\section{Additional Research and Development}

In this section, reference is made to programs which are important for heavy ion accelerators and which have not been discussed earlier.

\section{Ion source}

- "Common to all the accelerator systems which have been considered is the need for an effective and flexible ion source which can provide intense beams of a wide variety of ions. Cyclotrons and linacs require positive ion sources, while the electrostatic tandem accelerators employ a negative ion source. Positive ion sources, which will satisfy the required design criteria, are not available at the present time. It is, for example, not equally simple to make ion sources for all the various ions of interest. It is not possible to generate ions whose charge state is sufficiently high for many cases. It is, in fact, important to maximize the charge state in all cases, since the greater the charge state the greater is the maximum heavy ion energy.* Finally, we note that the lifetime of these sources is quite short. Bock of Darmstadt estimates lifetimes of the order of hours, while the GANIL group estimates the lifetime is $20-40$ hours for this mode of operation.

Regarding negative ion sources, very encouraging developments have recently been made at the University of Pennsylvania where a sputtering technique has been employed. Ample beam currents of singly charged negative ions have been obtained and the technique seems to be successful with nearly all elements. However, difficulties remain for the noble gases, with the exception of $\mathrm{He}^{-}$.

A thorough discussion of this problem will be presented in the near future by the Panel on Ion Sources of the Committee on Nuclear Science. We, therefore, here simply emphasize that the performance of the ion source is critical for the performance of the accelerator complex and, for this reason, the Panel strongly recommends encouragement of ion source research and development.

* Present day sources can at best, and only for a few elements, produce heavy ions with charges in the range $8+$ to $12+$. 


\section{Beam pulsing}

The accelerator systems considered in this report all involve one or more rf accelerators. For good energy resolution, it is necessary to inject the beam into such accelerators at the appropriate phase of the rf cycle. This requires the ability to inject short, intense beam bursts correctly phased with the rf frequency. The means by which the required performance of the injecting accelerator plus ion source will be obtained has not been given detailed attention. The Panel recommends further detailed study of this problem with sufficient resources to allow firm definition of adequate solutions in the near future.

\section{Multiple beams}

All of the proposed accelerator designs provide a single beam of a single ion species with a given energy at a given time. This limits the number of experiments which can be simultaneously performed within a given time to essentially one. It would be a more effective use of the accelerator and would insure more rapid progress in the field if it were possible to perform several experiments in parallel. The Panel recommends active consideration of how such sharing might be effectively implemented.

\section{ERA concept}

The electron ring accelerator involves the acceleration of a structure consisting of a heavy ion trapped in a ring composed of electrons by a magnetic field. There has been substantial progress in recent years in this field. Rings have been produced and have been accelerated. However, many important questions remain before the mechanisms involved in the formation and acceleration process are understood. We are thus very far from a stage in which an accelerator involving the ERA concept can be designed. Clearly, additional research is required before a realistic appraisal can be made. 


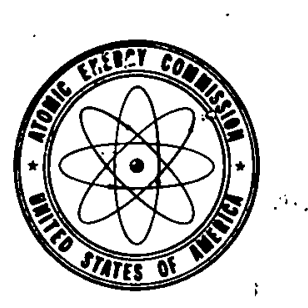

\section{UNITED STATES \\ ATOMIC ENERGY COMMISSION}

WASHINGTON, D.C. 20545

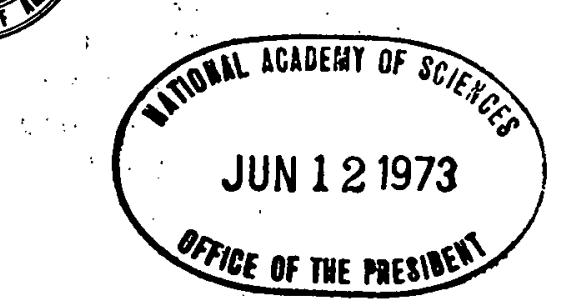

JUN 121973

HAND DELIVERED

Dr. Philip Handler, President

National Academy of Sciences

2101 Constitution Avenue

Washington, D. C. 20418

Dear Dr. Handler:

Recent surveys by the AEC and others, including the National Academy of Sciences, have discussed the interdisciplinary nature of heavy-ion research activities. It has been concluded that many opportunities in several scientific disciplines, including especially nuclear physics and nuclear chemistry, could be beneficially exploited through use of heavy-ion beams. As is pointed out by the Academy in its "Physics in Perspective" report, heavy-ion research "... makes accessible for the first time entirely new modes of nuclear motion and dynamics and permit study of more familiar phenomena in entirely new regions of angular momentum and other parameters. It also makes accessible new nuclear species -- both through moving away from the nuclear valley of stability to isotopes as yet unknown and upward along this valley to possible new super-transuranic elements..." The Academy report further recommended "To maintain the momentum already built up in this field, a national installation...should be initiated as soon as possible (which) would greatly extend the capabilities of the superHILAC or the other facilities now beginning exploration in this field."

It is clear that interest in heavy-ion research is growing throughout the scientific community, and that immediate attention must be given to future capabilities so that full benefits may be derived from this dynamic and promising new field.

It would be appreciated if the National Academy of Sciences would conduct a survey of the heavy-ion field so as to put recent developments into clearer perspective. It is our belief that such a survey would enable us to refine our planning and identify clear cut alternatives vis-a-vis near-term goals and objectives. We would hope that a study of such magnitude could be completed in approximately two or three months. 
We would like the Academy to undertake a detailed technical assessment aimed at providing the following information: 1) a quantitative determination of additional experimental facilities needed, including size and scope of ancillary laboratory facilities as well as accelerators, from the viewpoint of the total national program outlined in the above referenced documents; 2) from an evaluation of accelerator technology applicable for heavy-ion acceleration, a determination, if a new national facility should be deemed necessary, of what accelerator technology should be adopted; and 3) identification and study of any alternatives to the above which would meet the national need in heavyion research.

We would be happy to discuss this matter with you or Mr. Coleman in more detail and work with you in developing a plan that would form a basis for initiation of work and execution of a contract.

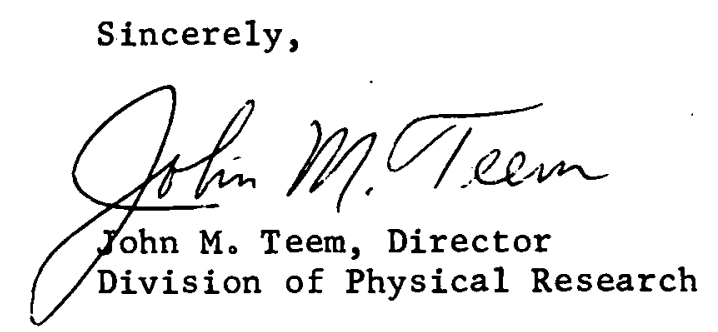

